\title{
Geographic analysis of Red List Rhododendron (Ericaceae) taxa by country of origin identifies priorities for ex situ conservation
}

\author{
M.B. MacKay ${ }^{1}$, S.E. Gardiner ${ }^{2}$
}

\section{Key words}

botanical gardens

Malesian flora

plant collections

Target 8

threatened species

\begin{abstract}
A Red List assessment is insufficient to determine priorities for ex situ conservation in large genera such as Rhododendron, where there may be hundreds of taxa in any one Red List category. We have utilised an analysis of the geographic origins of 1215 taxa of Rhododendron (Ericaceae) as a method to prioritise Red List taxa for ex situ conservation. This analysis includes descriptions of distribution and endemism by country of origin, analysis of the incidence of the 715 Red List taxa by country of origin, and determination of the extent to which taxa from each country of origin are in cultivation. We determined that of 30 countries of origin and a 'Europe' aggregate, 24 origins contain Red List taxa. Of those 24 origins, 17 origins and 'Europe' have greater than $75 \%$ of Red List taxa 'in cultivation', as defined in this study, so that Target 8 of the Global Strategy for Plant Conservation has theoretically been met. However, for some of these origins the number of each taxon held 'in cultivation' is very low and genetic diversity is likely to be poor. The remaining six countries of origin have less than $75 \%$ of Red List Rhododendron taxa recorded 'in cultivation' (Indonesia (28 \%), Papua New Guinea (29 \%), Malaysia (59 \%), China (60 \%), Japan $(62 \%)$ and Solomon Islands $(0 \%))$. Analysis of a set of Red List factors and 'not in cultivation' factors reveals that Red List taxa from Indonesia, China and Papua New Guinea should take priority for ex situ conservation.
\end{abstract}

Published on 15 August 2017

\section{INTRODUCTION}

Determination of priorities is one of the key challenges of conservation decision making and underpins successful development of both in situ and ex situ conservation strategies (Oldfield 2010, Ma et al. 2013). Under the Global Strategy for Plant Conservation (GSPC) (Sharrock et al. 2014) priorities are initially driven by the Red List process, conducted as part of Target 2 of the Strategy, whereby taxa under threat are identified and prioritised ahead of remaining taxa. Target 2 then drives Target 8, which requires $75 \%$ of Red List taxa to be in cultivation by 2020 (Sharrock 2012). This process appears to be sufficient for small genera comprised of similar life forms, but does not have a fine enough resolution for large genera of wide distribution and multiple life forms, where there may be hundreds of taxa in any one Red List category. In these genera additional factors such as taxonomic groupings, geographic representation, endemism and centres of diversity (Farnsworth et al. 2006, Castaneda-Alvarez et al. 2015, Cavendar et al. 2015), can be used to identify key groups of taxa. Rhododendron L. (Ericaceae) is one such large genus (of about 1215 taxa) in which Gibbs et al. (2011) and Argent (2015) Red Listed 715 taxa (MacKay \& Gardiner 2017). In an earlier study we focussed on determination of ex situ conservation priorities at the subgenus level ${ }^{3}$ (MacKay \& Gardiner 2017), thereby laying the foundation for the present study which examines another prioritisation mechanism. Here we examine a series of factors that can be used to identify taxa from geographic origins that should be prioritised for ex situ conservation.

\footnotetext{
1 Institute of Agriculture and Environment, Massey University, Private Bag 11-222, Palmerston North 4442, New Zealand;

corresponding author e-mail: m.b.mackay@massey.ac.nz.

2 The New Zealand Institute for Plant \& Food Research, Private Bag 11-600, Palmerston North 4442, New Zealand.
}

Rhododendron is a useful exemplar of the issues and challenges faced in ex situ conservation. Firstly, it illustrates the 'big genus' (Frodin 2004) characteristics of large size combined with taxonomic complexity and active speciation, which complicate conservation decision making (Ennos et al. 2005, 2012, Goodall-Copestake et al. 2005, Samain et al. 2009, Blackmore et al. 2011), and which prompted our examination of conservation priorities at the subgenus level (MacKay \& Gardiner 2017). Secondly, Rhododendron represents a microcosm of conservation issues because it encompasses a wide range of life forms, ecological niches and habitats. The genus is centred in Asia and Southeast Asia and is one of the largest plant genera in China (Lopez-Pujol et al. 2006) and the Southeast Asian floristic region (Van Welzen et al. 2005, Webb \& Ree 2012), with only about 40 of 1215 taxa originating in Europe and North America. The distribution in Asia stretches from Pakistan in the west, through India and the Himalaya (Nepal, Bhutan and Myanmar) to China and eastern Russia, and to the islands of Japan and Taiwan. Moving southeast the distribution encompasses Thailand, Vietnam, the islands of Malaysia, Indonesia, the Philippines and Papua New Guinea as far east as Solomon Islands, and south to Australia. The distribution coincides with biodiversity hotspots in Sikkim (Pradhan et al. 2015), Indo-Burma (Krupnick \& Kress 2003), Yunnan, Sichuan and Taiwan (Lopez-Pujol et al. 2006), the mountains of southwest China (Sharrock et al. 2014, Liu et al. 2015), Malaysia (Sharrock et al. 2014, Van der Ent et al. 2015), Sundaland, the Philippines and Wallacea (Krupnick \& Kress 2003) and New Guinea (Melick et al. 2012, Sharrock et al. 2014).

\footnotetext{
3 The 1215 Rhododendron taxa are divided into nine subgenera: Azaleastrum 37 taxa, Candidastrum 1 taxon, Hymenanthes 358 taxa, Mumeazalea 1 taxon, Pentanthera 34 taxa, Rhododendron 258 taxa, Therorhodion 2 taxa, Tsutsusi 124 taxa and Vireya 400 taxa.
} 
Rhododendron inhabits lowland to alpine zones within a range of climate zones, from subarctic tundra to tropical rainforest, to tropical-zone mountaintops. Rhododendrons grow in swamps, meadows, grasslands, on mountainsides, on rocks and cliffs, and in ravines and river valleys (Cox \& Hutchison 2008, Gibbs et al. 2011, Argent 2015). Many taxa are forest species, either trees or shrubs, and can be either a major or dominant component of the forest (Maren \& Vetaas 2007, Shi \& Zhu 2009, Bharali et al. 2011, Paudel et al. 2012). Some species form pure stands (Paudel et al. 2012, Ranjitkar et al. 2014) or are keystone species (Singh et al. 2009, Baird et al. 2014, Bharali et al. 2014). The epiphytic habit is common in some taxonomic groups, particularly in subg. Vireya (Argent 2015). Many are high altitude plants that are key species at, or on either side of, the tree line (Paul et al. 2005, Singh et al. 2009, Paudel et al. 2012). Above the tree line shrubby or creeping rhododendrons occur in shrub associations or in alpine meadows (Cox \& Hutchison 2008, Paudel et al. 2012).

Rhododendron taxa have a range of economic and cultural values. Rhododendron niveum Hook.f. is the state tree of Sikkim (Pradhan et al. 2015), while some species are the focus of tourist attractions (Maren \& Vetaas 2007, Mao \& Gogoi 2012). Various taxa provide ingredients for liquor, fragrance or incense (Singh et al. 2009) and pharmaceutical products (Popescu \& Kopp 2013). Many taxa are grown as ornamental plants in their own right (Cox \& Cox 1997) or have been hybridised to develop horticultural cultivars (Leslie 2004). Several species support communities through use as firewood (Paul et al. 2005), although overharvesting of firewood is also a threat to survival of some taxa (Singh et al. 2009, Pradhan et al. 2015). Other threats include grazing and cropping, forest clearance, logging, habitat degradation, land development, infrastructure construction, increasing tourism leading to resort development in wilderness areas, illegal collecting, excessive collecting of species used for medicinal purposes, pollution, and climate change (Paul et al. 2005, Maren \& Vetaas 2007, Singh et al. 2009, Oldfield 2010, Gibbs et al. 2011, Hird 2012, Ma et al. 2013, Liu et al. 2015, Pradhan et al. 2015, Van der Ent et al. 2015).

Although plant conservation involves a range of initiatives, in this study we focus on ex situ conservation where taxa are held in living collections in botanic gardens. This is an integral component of an overall conservation programme (Heywood 2015) and is fundamental to the achievement of Target 8 of the GSPC (Sharrock et al. 2014). Target 8 can be a challenge for mega-diverse countries such as China (Raven 2011) and India (Jalli et al. 2015) and is made more difficult by a mismatch between location of botanic gardens and areas of greatest diversity (Sharrock et al. 2014, Huang et al. 2015). Other challenges for ex situ conservation include accurate identification of specimens, appropriate documentation of collections, development of collections from wild-source material, acquisition of Red List taxa rather than common taxa, securing sufficient accessions at enough sites, and achieving adequate genetic representation (Lopez-Pujol et al. 2011, Pritchard et al. 2011, Rae 2011, Kozlowski et al. 2012, Cires et al. 2013, Ensslin et al. 2015, Huang et al. 2015). Of particular concern is insufficient capacity to house every rare taxon in a botanical collection (Heywood 2009, Lopez-Pujol et al. 2011, Ma et al. 2014), indicating that robust processes are needed to identify ex situ conservation priorities.

In addition to completion of a Red List assessment, planning for Target 8 requires two key resources; data on the diversity and frequency of taxa already in cultivation (Cires et al. 2013) and, for large genera, additional mechanisms to sort taxa into groups of higher and lower urgency, respectively. In comparison with threatened plants overall $(29 \%$ of species in cultivation or seed banks (Sharrock et al. 2014)) Rhododendron is relatively well placed with $70 \%$ of 'all taxa' and $56 \%$ of Red List taxa held in living collections (MacKay \& Gardiner 2017) small increase from the $67 \%$ and $53 \%$ reported by Botanic Gardens Conservation International (BGCl) in 2012 (BGCI 2012). However, while the overall figure is encouraging, our earlier study determined that no subgenus reached Target 8 and some subgenera are very poorly represented in cultivation (MacKay \& Gardiner 2017).

The objective of the present study was to extend the previous study by examining Rhododendron and its Red List taxa in relation to country of origin. Our analysis included:

i. description of the origins and endemism of Rhododendron taxa in general and Red List taxa in particular;

ii. use of a Red List analysis to identify those countries with the most acute conservation issues;

iii. description of the extent to which taxa from each country are in cultivation;

iv. application of a 'not in cultivation' analysis to identify those countries whose taxa are poorly represented in cultivation;

v. combination of the analyses to identify which countries should take priority for ex situ conservation; and

vi. proposal of elements and geographical priorities for subsequent conservation action.

\section{METHODS}

\section{Data-set}

Our data-set was originally constructed for a study of conservation priorities in Rhododendron at subgenus level (MacKay \& Gardiner 2017) and was re-developed for the present geographical analysis. The data-set comprised 1215 taxa and their Red List assessments (Gibbs et al. 2011, Argent 2015), with 'Red List taxa' (715 taxa) defined as those in all categories except Least Concern, while the remaining 500 taxa are in the Least Concern category. Taxa included species, subspecies and varieties as listed by Argent (2015) for subg. Vireya and Gibbs et al. (2011) for the other eight subgenera, with taxonomy checked and organised according to Chamberlain et al. (1996), Fang et al. (2005) and Argent (2015). International data on taxa in cultivation were extracted in 2015 from the BGCl online database (https://www.bgci.org/plant_search. php; acc. 9-10 Oct. 2015) and used as a primary indicator of taxa in cultivation (the BGCl database has 1363723 entries of 496775 taxa from 1147 botanic gardens world-wide (bgci. org; acc. 2 May 2016)). Additional data on taxa in cultivation were added from the online databases at Royal Botanic Garden Edinburgh (Catalogue of the Living collections: http:// elmer.rbge.org.uk/bgbase/livcol/bgbaselivcol.php; acc. 9-10 Oct. 2015) and Royal Botanic Garden Kew (Electronic Plant Information Centre: Living Collections. http://epic.kew.org/ searchepic/searchpage.do; acc. 29 Oct. 2015), which were identified as the largest collections world-wide (BGCI 2012), as well as from New Zealand collections that contain a substantial range of Rhododendron taxa (MacKay et al. 2017). Presence or absence of wild-source material was noted for each taxon. Taxa were defined as 'in cultivation' if they were recorded in any of the collections investigated. (Note that we use the terms 'collection' or 'collections' to refer to an assemblage of living plants on a site such as a botanic garden.)

\footnotetext{
${ }^{4}$ Of the 1215 taxa assessed by Gibbs et al. (2011) and Argent (2015), the combined Red List results were; 2 taxa Extinct, 1 taxon Extinct in the Wild, 36 taxa Critically Endangered, 39 taxa Endangered, 260 taxa Vulnerable, 62 taxa Near Threatened, 315 taxa Data Deficient, and 500 taxa Least Concern (MacKay \& Gardiner 2017).
} 


\section{Geographical assignment and degree of endemism}

For the geographical analysis each taxon was assigned to countries of origin according to Gibbs et al. (2011) and Argent (2015); those taxa with a single country of origin were coded as endemic. All countries were treated as separate data categories, except for those in Europe which were aggregated as 'Europe', as these 24 countries are home to only 10 taxa (eight taxa confined to this region and two circumpolar taxa that are shared with Asia and North America). This approach was taken to simplify the analysis and to reduce the size of tables.

Data were analysed in a number of ways, beginning with patterns of distribution and endemism. Firstly, the numbers of taxa, endemic taxa and non-endemic taxa were determined for each country of origin. The extent of sharing of non-endemic taxa was quantified and the numbers of taxa shared with other countries organised into bands of ' $50 \%$ or more of non-endemic taxa shared' followed by $25-49 \%$, less than $25 \%$ and countries with which single taxa are shared. (Countries may share different single taxa with different countries, e.g., China shares just Rhododendron moulmainense Hook.f. with Cambodia, while it shares only Rhododendron lapponicum (L.) Wahlenb. with Canada.) Secondly, the number of Red List taxa in the various Red List categories was determined for each country and then repeated for endemic taxa.

\section{Derivation of a 'Red List' score}

Ranking methods are a useful way to assign priorities (Krupnick \& Kress 2003, Farnsworth et al. 2006, Rahman 2015, MacKay \& Gardiner 2017) and in our third analysis each country was ranked for eight Red List factors and the rankings used to derive a Red List score. The eight factors used in the ranking were: number of Red List taxa; percentage of taxa Red Listed; number of Red List taxa that are endemic; percentage of Red List taxa that are endemic; number of Red List taxa rated Data Deficient; percentage of Red List taxa rated Data Deficient; number of Data Deficient taxa that are endemic; and percentage of Data Deficient taxa that are endemic. This ranking had 21 ranks with the top-ranked country scoring 21 points, the next 20 points, etc.; each country was ranked for each of the eight factors, then the ranking scores for each country were summed to generate a 'Red List score' (maximum score 168). Countries with the highest score were assigned highest priority for conservation.

\section{Security 'in cultivation' and derivation of a 'not in cultivation' score'}

The fourth analysis examined the extent to which taxa are 'in cultivation' by describing the numbers and percentages of 'all taxa' (Least Concern taxa + Red List taxa), and Red List taxa separately that are 'in cultivation' for each country. The average number of BGCI records per taxon for groups of 'all taxa' and Red List taxa for each country were calculated by dividing the number of records for the group by the number of taxa 'in cultivation' in that group, thereby giving a measure of the security of those taxa that are 'in cultivation'. Any average fewer than three records per taxon is considered a risk threshold (Lowe 1988) below which taxa are not secure in cultivation.

The fifth analysis involved derivation of a 'not in cultivation' score using a series of nine 'not in cultivation' factors, as absence of Red List taxa from collections is also a driver of conservation priorities (Farnsworth et al. 2006, Castaneda-Alvarez et al. 2015). Eight of these factors had a similar form to the Red List analysis and were the numbers and percentages of taxa 'not in cultivation' for Red List taxa, endemic Red List taxa, Data Deficient taxa, and endemic Data Deficient taxa. The ninth factor was a rounded average number of records per Red List taxon on the $\mathrm{BGCl}$ database; this was calculated by dividing the number of records for each country by the total number of taxa for that country (not just the number 'in cultivation'), thereby giving a measure of the extent to which that group is represented 'in cultivation'. This ranking had 15 ranks so the highest ranked country scored 15 points, the next ranked country 14 points, and so on. The ranking scores for the nine factors for each country were summed to derive the 'not in cultivation' score (maximum score 135), with the highest scoring countries exhibiting the poorest representation of Red List taxa 'in cultivation' and therefore having the greatest urgency for ex situ conservation.

\section{Development of Total Score and implication for conservation}

In the sixth step we ranked origins according to a Total Score in order to identify those origins with the combined problem of an acute conservation issue and poor representation of Red List taxa 'in cultivation'. The analysis involved summing of the Red List score and 'not in cultivation' score to generate a Total Score for each country of origin (maximum score 303 points). The final step was to propose conservation actions and priorities for Rhododendron based on this final ranking.

\section{RESULTS}

\section{Patterns of origin and endemism}

The greatest number of Rhododendron taxa originate in China (649 taxa) followed by Indonesia (229), Myanmar (137), India (107) and Papua New Guinea (100). Seventeen countries and 'Europe' are each the origin of 10 or more taxa, while 13 countries are the origin of fewer than 10 taxa (Table 1). China is also the origin of the greatest number of endemic taxa (442 taxa), followed by Indonesia (168), Papua New Guinea (64), Japan (58) and Malaysia (53). Myanmar and India, while having large numbers of taxa, are relatively low in endemics (9 and 14, respectively); the Philippines, the United States of America (USA) and Taiwan have fewer taxa overall but have more endemic taxa (30, 21 and 16, respectively) than the two previous origins. In Australia and Sri Lanka, $100 \%$ of taxa are endemic (a total of three taxa), while high percentages of taxa are endemic in the Philippines (91\%), 'Europe' (80\%), Japan (78\%), Indonesia (73\%) and Taiwan (70\%). China (68\%), Malaysia (64 \%) and Papua New Guinea (64 \%) are mid-range for this measure.

Table 1 also details the sharing of non-endemic taxa with other countries. For example, more than 50 \% of non-endemic Chinese taxa are also found in Myanmar and India, while another 12 countries share $25 \%$ or less of the taxa that originate in China. For Indonesian taxa, about half of the 61 non-endemic taxa are shared with each of Malaysia and Papua New Guinea. Many countries have single taxa (not always the same taxon) in common with other countries; these tend to be taxa that are widespread in Asia (Rhododendron simsii Planch., R. moulmainense) or the circumpolar taxa ( $R$. lapponicum, $R$. tomentosum (Stokes) Harmaja) that are found in the northernmost regions of Asia, Europe and North America.

\section{Red List taxa and endemic Red List taxa}

The greatest number of Red List taxa originate in China (447 Red List taxa), followed by Indonesia (111), Myanmar (62), India (45) and Papua New Guinea (34) (Table 2). The Red List categories Vulnerable or Data Deficient dominate in most countries; two exceptions are the Philippines and Japan which both have relatively high numbers of Critically Endangered taxa. While China is the origin of the greatest number of Critically Endangered taxa, this comprises only $3 \%$ of Red List taxa overall for that country whereas the Critically Endangered taxa comprise $19 \%$ of the Red List taxa for Japan and $32 \%$ 
Table 1 Countries of origin of Rhododendron (Gibbs et al. 2011, Argent 2015) ranked by number of taxa: showing number of taxa, number of endemic taxa, number of non-endemic taxa, and other countries with which the non-endemic taxa are shared. Total number of taxa is 1215 ; however, column two will not sum to 1215 as many taxa have more than one origin.

\begin{tabular}{|c|c|c|c|c|c|c|c|c|}
\hline $\begin{array}{l}\text { Country of } \\
\text { origin }\end{array}$ & o. of taxa & $\begin{array}{l}\text { No. of taxa } \\
\text { that are } \\
\text { endemic }\end{array}$ & $\begin{array}{l}\text { Percentage of } \\
\text { taxa that } \\
\text { are endemic }\end{array}$ & $\begin{array}{l}\text { No. of non- } \\
\text { endemic taxa }\end{array}$ & $\begin{array}{l}\text { Countries (and no. } \\
\text { of taxa) with which } \\
50 \% \text { or more of } \\
\text { non-endemic taxa } \\
\text { are shared }\end{array}$ & $\begin{array}{l}\text { Countries (and no. } \\
\text { of taxa) with which } \\
25-49 \% \text { of non- } \\
\text { endemic taxa are } \\
\text { shared }\end{array}$ & $\begin{array}{l}\text { Countries (and no. } \\
\text { of taxa) with which } \\
\text { less than } 25 \% \text { of } \\
\text { non-endemic taxa } \\
\text { ar shared }\end{array}$ & $\begin{array}{l}\text { Countries with } \\
\text { which one taxon is } \\
\text { shared (not always } \\
\text { the same taxon) }\end{array}$ \\
\hline China & 649 & 442 & $68 \%$ & 207 & $\begin{array}{l}\text { Myanmar } 124 \\
\text { India } 88\end{array}$ & & $\begin{array}{l}\text { Bhutan } 49 \\
\text { Nepal } 30 \\
\text { Vietnam } 28 \\
\text { Japan } 8 \\
\text { Korea Nth } 6 \\
\text { Taiwan } 6 \\
\text { Hong Kong } 6 \\
\text { Thailand } 5 \\
\text { Laos } 5 \\
\text { Russia: east } 5 \\
\text { Korea Sth } 4 \\
\text { Mongolia } 3\end{array}$ & $\begin{array}{l}\text { Cambodia } \\
\text { Malaysia } \\
\text { 'Europe' } \\
\text { Canada } \\
\text { Greenland }\end{array}$ \\
\hline Indonesia & 229 & 168 & $73 \%$ & 61 & PNG 34 & Malaysia 25 & $\begin{array}{l}\text { Brunei } 9 \\
\text { Philippines } 3 \\
\text { Thailand } 2\end{array}$ & \\
\hline Myanmar & 137 & 9 & $7 \%$ & 128 & China 124 & India 41 & $\begin{array}{l}\text { Bhutan } 13 \\
\text { Vietnam } 12 \\
\text { Nepal } 10 \\
\text { Thailand } 8 \\
\text { Laos } 4 \\
\text { Japan } 2 \\
\text { Hong Kong } 2 \\
\text { Taiwan 2 }\end{array}$ & $\begin{array}{l}\text { Bangladesh } \\
\text { Malaysia } \\
\text { Cambodia }\end{array}$ \\
\hline India & 107 & 14 & $13 \%$ & 93 & $\begin{array}{l}\text { China } 88 \\
\text { Bhutan } 49\end{array}$ & $\begin{array}{l}\text { Myanmar } 41 \\
\text { Nepal } 30\end{array}$ & $\begin{array}{l}\text { Vietnam } 7 \\
\text { Thailand } 5 \\
\text { Laos } 2 \\
\text { Bangladesh } 2\end{array}$ & $\begin{array}{l}\text { Hong Kong } \\
\text { Japan } \\
\text { Cambodia } \\
\text { Malaysia } \\
\text { Taiwan }\end{array}$ \\
\hline $\begin{array}{l}\text { Papua New } \\
\text { Guinea (PNG) }\end{array}$ & 100 & 64 & $64 \%$ & 36 & Indonesia 34 & & Solomons 2 & \\
\hline Malaysia & 83 & 53 & $64 \%$ & 30 & Indonesia 25 & Brunei 12 & Thailand 4 & $\begin{array}{l}\text { India } \\
\text { Hong Kong } \\
\text { Cambodia } \\
\text { Laos } \\
\text { Vietnam } \\
\text { Burma } \\
\text { Japan } \\
\text { Taiwan }\end{array}$ \\
\hline Japan & 74 & 58 & $78 \%$ & 16 & $\begin{array}{l}\text { Russia: east } 8 \\
\text { China } 8\end{array}$ & $\begin{array}{l}\text { Korea Sth } 7 \\
\text { Korea Nth } 6\end{array}$ & $\begin{array}{l}\text { Mongolia } 3 \\
\text { Hong Kong } 3 \\
\text { Myanmar } 2 \\
\text { Taiwan } 2 \\
\text { Thailand } 2 \\
\text { Laos } 2\end{array}$ & $\begin{array}{l}\text { Cambodia } \\
\text { Vietnam } \\
\text { India } \\
\text { Malaysia } \\
\text { 'Europe' } \\
\text { Canada } \\
\text { Greenland } \\
\text { USA }\end{array}$ \\
\hline Bhutan & 57 & 4 & $7 \%$ & 53 & $\begin{array}{l}\text { China } 49 \\
\text { India } 49\end{array}$ & $\begin{array}{l}\text { Nepal } 29 \\
\text { Myanmar } 13\end{array}$ & $\begin{array}{l}\text { Thailand } 2 \\
\text { Vietnam } 2\end{array}$ & Bangladesh \\
\hline Vietnam & 40 & 10 & $25 \%$ & 30 & China 28 & Myanmar 12 & $\begin{array}{l}\text { India } 7 \\
\text { Thailand } 5 \\
\text { Laos } 5 \\
\text { Bhutan } 2\end{array}$ & $\begin{array}{l}\text { Hong Kong } \\
\text { Cambodia } \\
\text { Malaysia } \\
\text { Taiwan } \\
\text { Japan } \\
\text { Sumatra }\end{array}$ \\
\hline Nepal & 34 & 3 & $9 \%$ & 31 & $\begin{array}{l}\text { India } 30 \\
\text { China } 30 \\
\text { Bhutan } 29\end{array}$ & Myanmar 10 & & Bangladesh \\
\hline Philippines & 33 & 30 & $91 \%$ & 3 & $\begin{array}{l}\text { Indonesia } 3 \\
\text { Malaysia } 3\end{array}$ & & & \\
\hline $\begin{array}{l}\text { United States } \\
\text { of America (USA) }\end{array}$ & 29 & 21 & $72 \%$ & 8 & Canada 7 & & & $\begin{array}{l}\text { Greenland } \\
\text { Japan } \\
\text { Russia: east }\end{array}$ \\
\hline Taiwan & 23 & 16 & $70 \%$ & 7 & $\begin{array}{l}\text { China } 6 \\
\text { Japan } 4\end{array}$ & $\begin{array}{l}\text { Vietnam } 2 \\
\text { Hong Kong } 2 \\
\text { Laos } 2 \\
\text { Thailand } 2\end{array}$ & & $\begin{array}{l}\text { India } \\
\text { Cambodia } \\
\text { Myanmar } \\
\text { Malaysia }\end{array}$ \\
\hline
\end{tabular}


Table 1 (cont.)

\begin{tabular}{|c|c|c|c|c|c|c|c|c|}
\hline $\begin{array}{l}\text { Country of } \\
\text { origin }\end{array}$ & No. of taxa & $\begin{array}{l}\text { No. of taxa } \\
\text { that are } \\
\text { endemic }\end{array}$ & $\begin{array}{l}\text { Percentage of } \\
\text { taxa that } \\
\text { are endemic }\end{array}$ & $\begin{array}{l}\text { No. of non- } \\
\text { endemic taxa }\end{array}$ & $\begin{array}{l}\text { Countries (and no. } \\
\text { of taxa) with which } \\
50 \% \text { or more of } \\
\text { non-endemic taxa } \\
\text { are shared }\end{array}$ & $\begin{array}{l}\text { Countries (and no. } \\
\text { of taxa) with which } \\
25-49 \% \text { of non- } \\
\text { endemic taxa are } \\
\text { shared }\end{array}$ & $\begin{array}{l}\text { Countries (and no. } \\
\text { of taxa) with which } \\
\text { less than } 25 \% \text { of } \\
\text { non-endemic taxa } \\
\text { ar shared }\end{array}$ & $\begin{array}{l}\text { Countries with } \\
\text { which one taxon is } \\
\text { shared (not always } \\
\text { the same taxon) }\end{array}$ \\
\hline Brunei & 12 & 0 & 0 & 12 & $\begin{array}{l}\text { Malaysia } 12 \\
\text { Indonesia } 9\end{array}$ & & & \\
\hline Thailand & 12 & 1 & $8 \%$ & 11 & Myanmar 8 & $\begin{array}{l}\text { China } 5 \\
\text { India } 5 \\
\text { Vietnam } 4 \\
\text { Laos } 4 \\
\text { Malaysia } 3\end{array}$ & $\begin{array}{l}\text { Bhutan } 2 \\
\text { Taiwan } 2 \\
\text { Japan } 2 \\
\text { Hong Kong } 2 \\
\text { Indonesia } 2\end{array}$ & Cambodia \\
\hline Korea Sth & 11 & 2 & $18 \%$ & 9 & $\begin{array}{l}\text { Japan } 7 \\
\text { Korea Nth } 6\end{array}$ & $\begin{array}{l}\text { China } 4 \\
\text { Russia: east } 4\end{array}$ & Mongolia 2 & $\begin{array}{l}\text { ‘Europe' } \\
\text { Canada } \\
\text { Greenland }\end{array}$ \\
\hline Korea Nth & 9 & 0 & 0 & 9 & $\begin{array}{l}\text { Korea Sth } 6 \\
\text { China } 6 \\
\text { Japan } 6 \\
\text { Russia: east } 6\end{array}$ & Mongolia 2 & & \\
\hline $\begin{array}{l}\text { 'Europe' } \\
\text { aggregate }^{1}\end{array}$ & 10 & 8 & $80 \%$ & 2 & $\begin{array}{l}\text { Canada } 2 \\
\text { Greenland } 2 \\
\text { Russia: east } 2 \\
\text { China } 1 \\
\text { Mongolia } 1 \\
\text { Japan } 1 \\
\text { Korea Nth } 1 \\
\text { Korea Sth } 1\end{array}$ & & & $\begin{array}{l}\text { China } \\
\text { Mongolia } \\
\text { Japan } \\
\text { Korea Nth } \\
\text { Korea Sth }\end{array}$ \\
\hline Laos & 7 & 0 & 0 & 7 & $\begin{array}{l}\text { Vietnam } 6 \\
\text { China } 5 \\
\text { Thailand } 4\end{array}$ & Myanmar 3 & & $\begin{array}{l}\text { India } \\
\text { Hong Kong } \\
\text { Cambodia } \\
\text { Malaysia } \\
\text { Taiwan } \\
\text { Japan }\end{array}$ \\
\hline Hong Kong & 6 & 0 & 0 & 6 & $\begin{array}{l}\text { China } 6 \\
\text { Japan } 3\end{array}$ & $\begin{array}{l}\text { Thailand } 2 \\
\text { Taiwan } 2 \\
\text { Laos } 2\end{array}$ & & $\begin{array}{l}\text { Myanmar } \\
\text { India } \\
\text { Cambodia } \\
\text { Vietnam } \\
\text { Malaysia }\end{array}$ \\
\hline Canada & 9 & 0 & 0 & 9 & USA 7 & Greenland 3 & ‘Europe’ 2 & $\begin{array}{l}\text { China } \\
\text { Mongolia } \\
\text { Japan } \\
\text { Korea Nth } \\
\text { Korea Sth } \\
\text { Russia: east }\end{array}$ \\
\hline Mongolia & 5 & 0 & 0 & 5 & $\begin{array}{l}\text { Russia: east } 5 \\
\text { China } 3 \\
\text { Korea Nth } 3 \\
\text { Japan } 3\end{array}$ & Korea Sth 2 & & $\begin{array}{l}\text { ‘Europe' } \\
\text { Canada } \\
\text { Greenland }\end{array}$ \\
\hline Greenland & 3 & 0 & 0 & 3 & $\begin{array}{l}\text { Canada } 2 \\
\text { 'Europe' } 2 \\
\text { Russia: east } 2\end{array}$ & $\begin{array}{l}\text { China } 1 \\
\text { Mongolia } 1 \\
\text { Japan } 1 \\
\text { Korea Nth } 1 \\
\text { Korea Sth } 1\end{array}$ & & $\begin{array}{l}\text { China } \\
\text { Mongolia } \\
\text { Japan } \\
\text { Korea Nth } \\
\text { Korea Sth }\end{array}$ \\
\hline $\begin{array}{l}\text { Solomon Islands } \\
\text { (Solomons) }\end{array}$ & s & 0 & 0 & 2 & PNG 2 & & & \\
\hline Bangladesh & 2 & 0 & 0 & 2 & $\begin{array}{l}\text { Bhutan } 2 \\
\text { India } 2 \\
\text { China } 2 \\
\text { Myanmar } 1\end{array}$ & & & Myanmar \\
\hline Australia & 2 & 2 & $100 \%$ & 0 & & & & \\
\hline Afghanistan & 2 & 2 & 0 & 2 & Pakistan 2 & & & \\
\hline Pakistan & 2 & 2 & 0 & 2 & Afghanistan 2 & & & \\
\hline Sri Lanka & 1 & 1 & $100 \%$ & 0 & & & & \\
\hline Cambodia & 1 & 0 & 0 & 1 & $\begin{array}{l}\text { China1 } \\
\text { Hong Kong } 1 \\
\text { India } 1 \\
\text { Indonesia } 1 \\
\text { Laos } 1 \\
\text { Malaysia } 1 \\
\text { Myanmar } 1 \\
\text { Taiwan 1 } \\
\text { Thailand } 1 \\
\text { Vietnam } 1\end{array}$ & & & $\begin{array}{l}\text { China } \\
\text { Hong Kong } \\
\text { India } \\
\text { Indonesia } \\
\text { Laos } \\
\text { Malaysia } \\
\text { Myanmar } \\
\text { Taiwan } \\
\text { Thailand } \\
\text { Vietnam }\end{array}$ \\
\hline
\end{tabular}

1 'Europe' is defined as countries as far east as the easternmost extent of $R$. luteum and $R$. ponticum. This comprises 24 countries: Armenia, Azerbaydzhan, Austria, Bulgaria, Czechoslovakia, Finland, France, Germany, Denmark, Georgia, Italy, Moldova, Lebanon, Norway, Poland, Portugal, Russia: west (Abkhasiya, Dagestan, Osetiya), Slovenia, Spain, Sweden, Switzerland, Turkey, Ukraine, Yugoslavia. 
for the Philippines. Another exception to the general pattern is the reasonably high numbers of taxa in the Near Threatened category for China, Myanmar and India. Data Deficiency is most pronounced for taxa from China, Indonesia and Papua New Guinea.

The second entry for each origin in Table 2 shows the endemic taxa and their spread among the Red List categories. Taxa endemic to China exhibit a similar spread to Red List taxa overall; a pattern which is repeated for Indonesia, Malaysia, Papua New Guinea, Japan and the Philippines. Taxa from India demonstrate a different pattern; the 45 Red List taxa overall are dominated by Vulnerable and Near Threatened listings, while the 11 endemics are distributed almost evenly across the Critically Endangered, Endangered and Vulnerable categories. Although India does not have many endemic taxa they are in a relatively critical position; endemic taxa from Vietnam show a similar pattern. In contrast, the endemic Red List taxa from Myanmar and Bhutan are less threatened compared to Red List taxa overall from those countries.

\section{Ranking for Red List factors and 'Red List' score}

The Red List score (Fig. 1) was derived from the rankings for the eight Red List factors. Appendix 1 details these factors and shows that the top-ranked origins for numbers of Red List taxa are China (447 taxa) and Indonesia (111). The top-ranked origins for percentage of taxa Red Listed are Afghanistan and $\mathrm{Pa}-$ kistan (100 \% of the same two taxa), followed by China (69 \%), the Philippines (58 \%) and Vietnam (55\%). When number of endemic Red List taxa are considered, the top-ranked origins are China (354 taxa), followed by Indonesia (101), Papua New

Table 2 Countries of origin of Red List Rhododendron (Gibbs et al. 2011, Argent 2015) ranked by number of Red List taxa: showing numbers of taxa in each Red List category (first line), and numbers of endemic taxa in each Red List category (second line). Total number of Red List taxa is 715; however, column two will not sum to 715 as many taxa have more than one origin.

\begin{tabular}{|c|c|c|c|c|c|c|c|c|c|}
\hline \multirow[t]{2}{*}{ Country of origin } & \multirow[t]{2}{*}{$\begin{array}{l}\text { No. of Red } \\
\text { List taxa }\end{array}$} & \multirow[t]{2}{*}{$\begin{array}{l}\text { No. of Red } \\
\text { List taxa that } \\
\text { are endemic }\end{array}$} & \multicolumn{7}{|c|}{$\begin{array}{l}\text { Number of taxa in each Red List category. From left to right the categories are Extinct, } \\
\text { Extinct in the Wild, Critically Endangered, Endangered, Vulnerable, Near Threatened } \\
\text { and Data Deficient. }\end{array}$} \\
\hline & & & EX & EW & $\mathrm{CR}$ & EN & VU & NT & $\mathrm{DD}$ \\
\hline China & 447 & 354 & $\begin{array}{l}- \\
-\end{array}$ & $\begin{array}{l}- \\
-\end{array}$ & $\begin{array}{l}14 \\
12\end{array}$ & $\begin{array}{l}17 \\
15\end{array}$ & $\begin{array}{l}174 \\
123\end{array}$ & $\begin{array}{l}59 \\
38\end{array}$ & $\begin{array}{l}183 \\
166\end{array}$ \\
\hline Indonesia & 111 & 101 & - & $\begin{array}{l}- \\
-\end{array}$ & $\begin{array}{l}5 \\
5\end{array}$ & $\begin{array}{l}5 \\
5\end{array}$ & $\begin{array}{l}30 \\
26\end{array}$ & $\begin{array}{l}1 \\
1\end{array}$ & $\begin{array}{l}70 \\
64\end{array}$ \\
\hline Myanmar & 62 & 5 & $\begin{array}{l}- \\
-\end{array}$ & $\begin{array}{l}- \\
-\end{array}$ & $\begin{array}{l}2 \\
0\end{array}$ & $\begin{array}{l}2 \\
0\end{array}$ & $\begin{array}{r}33 \\
2\end{array}$ & $\begin{array}{r}15 \\
0\end{array}$ & $\begin{array}{r}10 \\
3\end{array}$ \\
\hline India & 45 & 11 & $\begin{array}{l}- \\
-\end{array}$ & $\begin{array}{l}- \\
-\end{array}$ & $\begin{array}{l}3 \\
3\end{array}$ & $\begin{array}{l}4 \\
4\end{array}$ & $\begin{array}{r}22 \\
3\end{array}$ & $\begin{array}{r}10 \\
0\end{array}$ & $\begin{array}{l}6 \\
1\end{array}$ \\
\hline Papua New Guinea & 34 & 29 & $\begin{array}{l}1 \\
1\end{array}$ & $\begin{array}{l}- \\
-\end{array}$ & $\begin{array}{l}- \\
-\end{array}$ & $\begin{array}{l}- \\
-\end{array}$ & $\begin{array}{l}6 \\
5\end{array}$ & - & $\begin{array}{l}27 \\
23\end{array}$ \\
\hline Malaysia & 32 & 25 & $\begin{array}{l}1 \\
1\end{array}$ & $\begin{array}{l}- \\
-\end{array}$ & $\begin{array}{l}1 \\
1\end{array}$ & $\begin{array}{l}4 \\
4\end{array}$ & $\begin{array}{l}18 \\
15\end{array}$ & $\begin{array}{l}1 \\
0\end{array}$ & $\begin{array}{l}7 \\
4\end{array}$ \\
\hline Japan & 26 & 25 & $\begin{array}{l}- \\
-\end{array}$ & $\begin{array}{l}- \\
-\end{array}$ & $\begin{array}{l}5 \\
5\end{array}$ & $\begin{array}{l}5 \\
5\end{array}$ & $\begin{array}{l}7 \\
7\end{array}$ & $\begin{array}{l}1 \\
0\end{array}$ & $\begin{array}{l}8 \\
8\end{array}$ \\
\hline Vietnam & 22 & 7 & $\begin{array}{l}- \\
-\end{array}$ & $\begin{array}{l}- \\
-\end{array}$ & $\begin{array}{l}1 \\
1\end{array}$ & $\begin{array}{l}2 \\
2\end{array}$ & $\begin{array}{l}7 \\
0\end{array}$ & $\begin{array}{l}4 \\
0\end{array}$ & $\begin{array}{l}8 \\
4\end{array}$ \\
\hline Philippines & 19 & 19 & - & - & $\begin{array}{l}6 \\
6\end{array}$ & $\begin{array}{l}1 \\
1\end{array}$ & $\begin{array}{l}5 \\
5\end{array}$ & - & $\begin{array}{l}7 \\
7\end{array}$ \\
\hline Bhutan & 13 & 1 & $\begin{array}{l}- \\
-\end{array}$ & $\begin{array}{l}- \\
-\end{array}$ & $\begin{array}{l}- \\
-\end{array}$ & $\begin{array}{l}- \\
-\end{array}$ & $\begin{array}{l}5 \\
0\end{array}$ & $\begin{array}{l}3 \\
0\end{array}$ & $\begin{array}{l}5 \\
1\end{array}$ \\
\hline Taiwan & 11 & 10 & - & $\begin{array}{l}1 \\
1\end{array}$ & $\begin{array}{l}- \\
-\end{array}$ & $\begin{array}{l}1 \\
1\end{array}$ & $\begin{array}{l}7 \\
7\end{array}$ & $\begin{array}{l}1 \\
0\end{array}$ & $\begin{array}{l}1 \\
1\end{array}$ \\
\hline USA & 8 & 7 & - & - & $\begin{array}{l}1 \\
1\end{array}$ & - & $\begin{array}{l}5 \\
5\end{array}$ & - & $\begin{array}{l}2 \\
1\end{array}$ \\
\hline Nepal & 4 & 1 & - & - & $\begin{array}{l}- \\
-\end{array}$ & - & $\begin{array}{l}2 \\
0\end{array}$ & - & $\begin{array}{l}2 \\
1\end{array}$ \\
\hline 'Europe' aggregate & 2 & 2 & $\begin{array}{l}- \\
-\end{array}$ & $\begin{array}{l}- \\
-\end{array}$ & $\begin{array}{l}- \\
-\end{array}$ & $\begin{array}{l}- \\
-\end{array}$ & $\begin{array}{l}2 \\
2\end{array}$ & $\begin{array}{l}- \\
-\end{array}$ & $\begin{array}{l}- \\
-\end{array}$ \\
\hline Pakistan & 2 & 0 & - & - & - & - & $\begin{array}{l}2 \\
0\end{array}$ & - & - \\
\hline Afghanistan & 2 & 0 & $\begin{array}{l}- \\
-\end{array}$ & - & - & - & $\begin{array}{l}2 \\
0\end{array}$ & - & - \\
\hline Russia: east & 1 & 1 & $\begin{array}{l}- \\
-\end{array}$ & $\begin{array}{l}- \\
-\end{array}$ & $\begin{array}{l}- \\
-\end{array}$ & $\begin{array}{l}- \\
-\end{array}$ & $\begin{array}{l}- \\
-\end{array}$ & - & $\begin{array}{l}1 \\
1\end{array}$ \\
\hline Brunei & 1 & 0 & - & - & $\begin{array}{l}- \\
-\end{array}$ & $\begin{array}{l}- \\
-\end{array}$ & $\begin{array}{l}- \\
-\end{array}$ & $\begin{array}{l}1 \\
0\end{array}$ & - \\
\hline Thailand & 1 & 1 & $\begin{array}{l}- \\
-\end{array}$ & $\begin{array}{l}- \\
-\end{array}$ & $\begin{array}{l}- \\
-\end{array}$ & $\begin{array}{l}- \\
-\end{array}$ & $\begin{array}{l}- \\
-\end{array}$ & - & $\begin{array}{l}1 \\
1\end{array}$ \\
\hline Laos & 1 & 0 & $\begin{array}{l}- \\
-\end{array}$ & $\begin{array}{l}- \\
-\end{array}$ & $\begin{array}{l}- \\
-\end{array}$ & $\begin{array}{l}- \\
-\end{array}$ & $\begin{array}{l}1 \\
0\end{array}$ & $\begin{array}{l}- \\
-\end{array}$ & $\begin{array}{l}- \\
-\end{array}$ \\
\hline Canada & 1 & 0 & $\begin{array}{l}- \\
-\end{array}$ & $\begin{array}{l}- \\
-\end{array}$ & $\begin{array}{l}- \\
-\end{array}$ & - & $\begin{array}{l}- \\
-\end{array}$ & $\begin{array}{l}- \\
-\end{array}$ & $\begin{array}{l}1 \\
0\end{array}$ \\
\hline Hong Kong & 1 & 0 & - & - & - & - & - & - & $\begin{array}{l}1 \\
0\end{array}$ \\
\hline Australia & 1 & 1 & - & - & - & - & $\begin{array}{l}1 \\
1\end{array}$ & - & - \\
\hline Solomon Islands & 1 & 0 & $\begin{array}{l}- \\
-\end{array}$ & $\begin{array}{l}- \\
-\end{array}$ & - & - & - & - & $\begin{array}{l}1 \\
0\end{array}$ \\
\hline
\end{tabular}




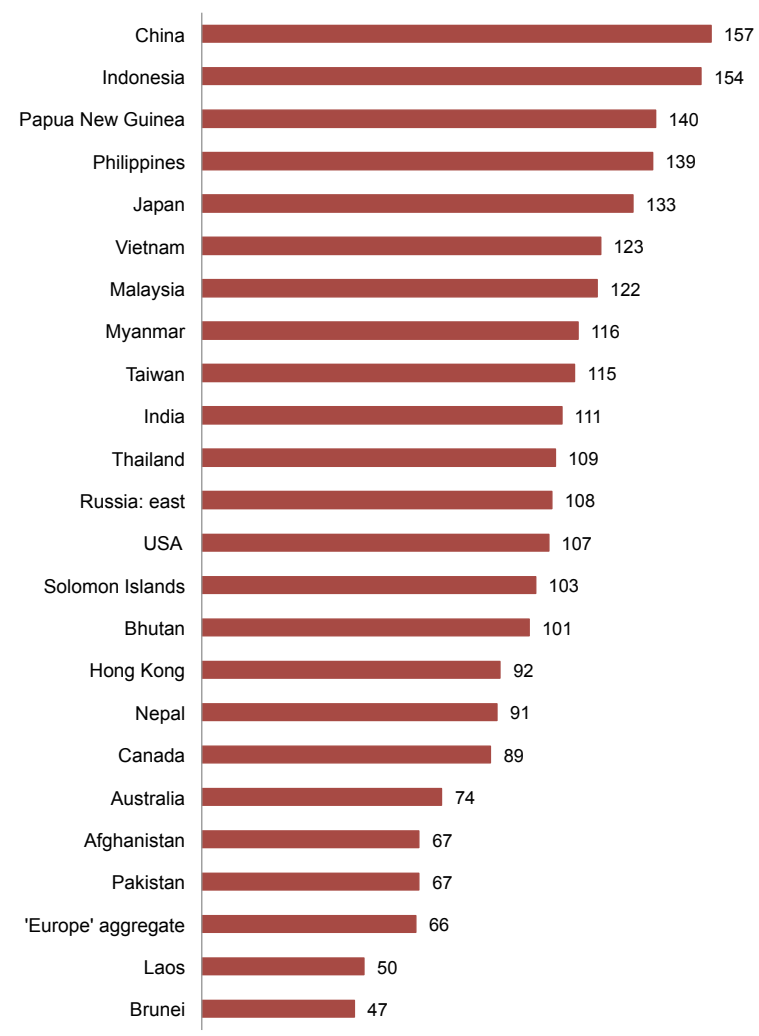

Fig. 1 Countries of origin of Rhododendron (Gibbs et al. 2011, Argent 2015) ranked according to Red List score (= sum of ranking scores for eight Red List factors, Appendix 1). Maximum score $=168$. The following origins have nil Red List taxa and therefore score zero on this ranking: Bangladesh, Cambodia, Greenland, Korea Nth, Korea Sth, Mongolia, Sri Lanka.

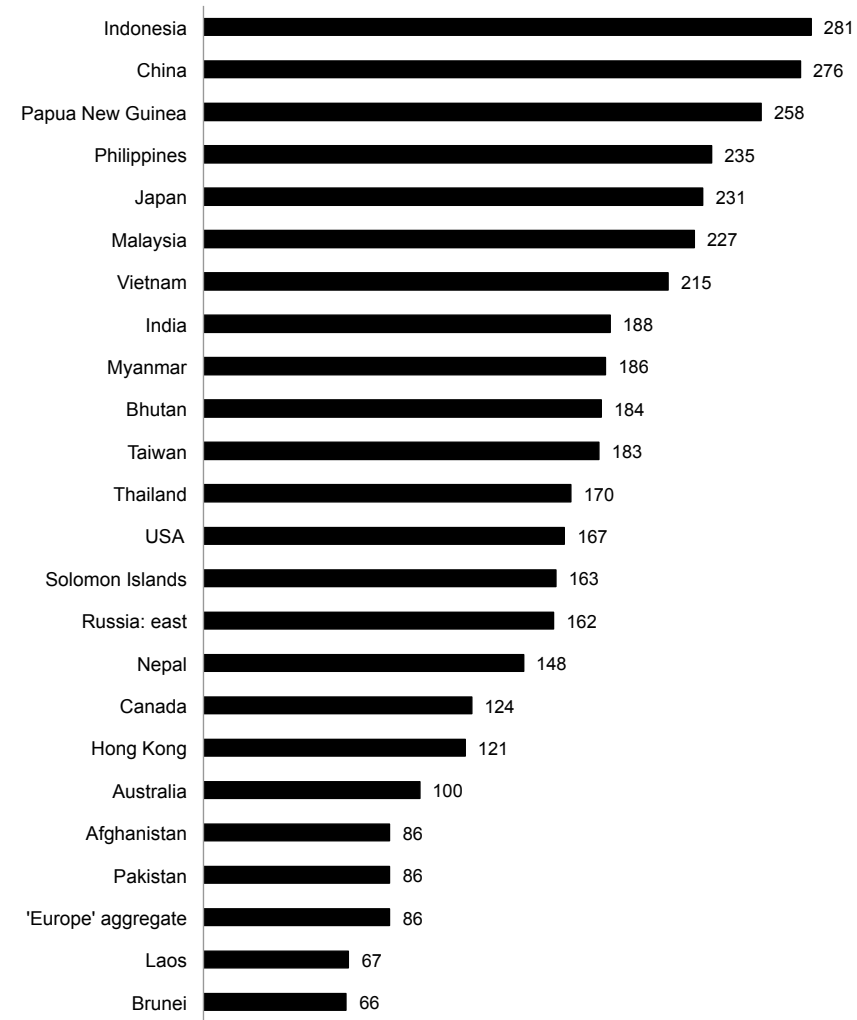

Fig. 3 Countries of origin of Rhododendron (Gibbs et al. 2011, Argent 2015) ranked according to Total Score (= Red List score, see Fig. 1, + 'not in cultivation' score, see Fig. 2). Maximum score $=303$. The following origins have nil Red List taxa and therefore score zero on this ranking: Bangladesh, Cambodia, Greenland, Korea Nth, Korea Sth, Mongolia, Sri Lanka.

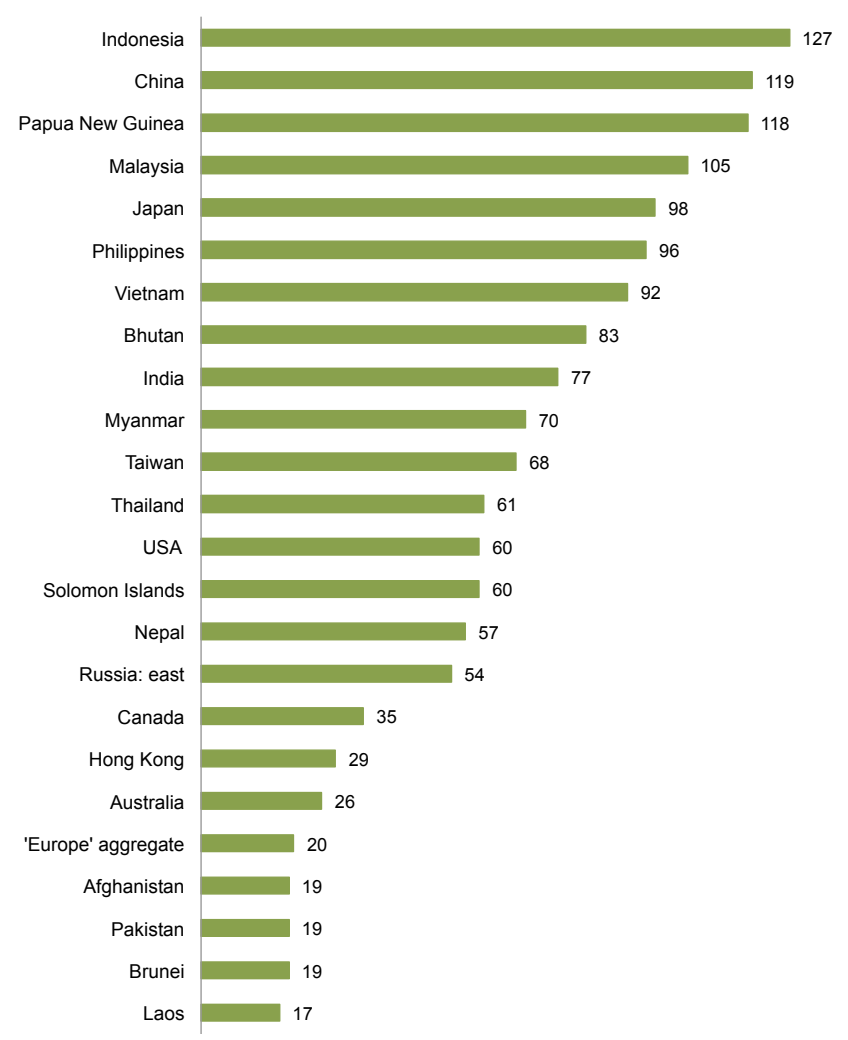

Fig. 2 Countries of origin of Rhododendron (Gibbs et al. 2011, Argent 2015) ranked according to 'not in cultivation' score (= sum of ranking scores for nine 'not in cultivation' factors, Appendix 2). Maximum score $=135$. The following origins have nil Red List taxa and therefore score zero on this ranking: Bangladesh, Cambodia, Greenland, Korea Nth, Korea Sth, Mongolia, Sri Lanka.

Guinea (29) and Malaysia and Japan (both with 25). For four origins and 'Europe', $100 \%$ of Red List taxa are endemic (the Philippines have 19 such taxa, with only 5 taxa in total from the remaining countries), and Japan has $96 \%$ of Red List taxa endemic. Other countries with high percentages of endemic Red List taxa are Indonesia and Taiwan (91\%) and the USA (88 \%).

For the number of Red List taxa rated Data Deficient, China tops the ranking (183 taxa), followed by Indonesia (70) and Papua New Guinea (27). The highest ranking for percentage of Red List taxa rated Data Deficient is held by several countries with $100 \%$ for this factor; however, there are only five taxa in total. Next in this ranking are Papua New Guinea (79 \%) and Indonesia (63\%). China, although it has 183 Data Deficient taxa, ranks 5 th for this factor, as the number of taxa is only $41 \%$ of the Red List taxa from that country.

When number of endemic Data Deficient taxa are considered, China again tops the ranking (166 taxa) and this is $91 \%$ of Data Deficient taxa from that country. The next rankings for number of Data Deficient taxa that are endemic go to Indonesia (64 taxa, $91 \%$ of Data Deficient taxa from that country) and Papua New Guinea (23 taxa, $85 \%$ of Data Deficient taxa from that country).

When the ranking scores (Appendix 1, column 1) are summed for each origin for the eight factors, the Red List score (Fig. 1) is led by China (157 points) followed by Indonesia (154 points), Papua New Guinea (140 points), the Philippines (139 points) and Japan (133 points).

\section{Taxa 'in cultivation'}

When the 844 'all taxa' and 400 Red List taxa 'in cultivation' (MacKay \& Gardiner 2017) are considered by country of origin, most origins have greater than $75 \%$ of 'all taxa' 'in cultivation' 
Table 3 Countries of origin of Rhododendron (Gibbs et al. 2011, Argent 2015) ranked by number of taxa: showing numbers and percentages of taxa and Red List taxa 'in cultivation', and average numbers of Botanic Gardens Conservation International (BGCl) records per taxon for 'all taxa' and Red List taxa that are 'in cultivation'. Average numbers of BGCI records = number of records/number of taxa 'in cultivation'. Total number of taxa is 1215 ; however, column two will not sum to 1215 as many taxa have more than one origin. Total number of Red List taxa is 715 ; however, column six will not sum to 715 as many taxa have more than one origin.

\begin{tabular}{|c|c|c|c|c|c|c|c|c|}
\hline Origin & No. of taxa & $\begin{array}{l}\text { No. of taxa } \\
\text { 'in cultivation' }\end{array}$ & $\begin{array}{l}\text { Percentage of } \\
\text { taxa } \\
\text { 'in cultivation' }\end{array}$ & $\begin{array}{l}\text { Average no. } \\
\text { of } \mathrm{BGCl} \\
\text { records per taxon } \\
\text { 'in cultivation' }\end{array}$ & $\begin{array}{l}\text { No. of Red } \\
\text { List taxa }\end{array}$ & $\begin{array}{l}\text { No. of Red } \\
\text { List taxa } \\
\text { 'in cultivation' }\end{array}$ & $\begin{array}{l}\text { Percentage of } \\
\text { Red List taxa } \\
\text { 'in cultivation' }\end{array}$ & $\begin{array}{l}\text { Average no. } \\
\text { of BGCI records } \\
\text { per Red List taxon } \\
\text { 'in cultivation' }\end{array}$ \\
\hline China & 649 & 464 & 72 & 14 & 447 & 269 & 60 & 9 \\
\hline Indonesia & 229 & 125 & 55 & 3 & 111 & 31 & 28 & 2 \\
\hline Myanmar & 137 & 126 & 92 & 15 & 62 & 56 & 90 & 10 \\
\hline India & 107 & 99 & 93 & 15 & 45 & 39 & 87 & 11 \\
\hline Papua New Guinea & 100 & 64 & 64 & 6 & 34 & 10 & 29 & 3 \\
\hline Malaysia & 83 & 65 & 78 & 4 & 32 & 19 & 59 & 2 \\
\hline Japan & 74 & 58 & 78 & 25 & 26 & 16 & 62 & 9 \\
\hline Bhutan & 57 & 52 & 91 & 18 & 13 & 11 & 85 & 14 \\
\hline Vietnam & 40 & 35 & 88 & 9 & 22 & 17 & 77 & 6 \\
\hline Philippines & 33 & 28 & 85 & 2 & 19 & 15 & 79 & 2 \\
\hline Nepal & 34 & 34 & 100 & 20 & 4 & 4 & 100 & 14 \\
\hline USA & 29 & 28 & 97 & 58 & 8 & 7 & 88 & 26 \\
\hline Taiwan & 23 & 20 & 87 & 19 & 11 & 9 & 82 & 18 \\
\hline Russia: east & 15 & 13 & 87 & 37 & 1 & 1 & 100 & 39 \\
\hline Brunei & 12 & 11 & 92 & 4 & 1 & 1 & 100 & 3 \\
\hline Thailand & 12 & 11 & 92 & 13 & 1 & 1 & 100 & 7 \\
\hline Korea Sth & 11 & 10 & 91 & 57 & 0 & $\mathrm{n} / \mathrm{a}$ & $\mathrm{n} / \mathrm{a}$ & $\mathrm{n} / \mathrm{a}$ \\
\hline 'Europe' aggregate & 10 & 10 & 100 & 67 & 2 & 2 & 100 & 70 \\
\hline Korea Nth & 9 & 8 & 89 & 69 & 0 & $\mathrm{n} / \mathrm{a}$ & $\mathrm{n} / \mathrm{a}$ & $\mathrm{n} / \mathrm{a}$ \\
\hline Canada & 9 & 9 & 100 & 39 & 1 & 1 & 100 & 1 \\
\hline Laos & 7 & 7 & 100 & 16 & 1 & 1 & 100 & 6 \\
\hline Hong Kong & 6 & 6 & 100 & 14 & 1 & 1 & 100 & 9 \\
\hline Mongolia & 5 & 5 & 100 & 39 & 0 & $\mathrm{n} / \mathrm{a}$ & $\mathrm{n} / \mathrm{a}$ & $\mathrm{n} / \mathrm{a}$ \\
\hline Greenland & 3 & 3 & 100 & 25 & 0 & $\mathrm{n} / \mathrm{a}$ & $\mathrm{n} / \mathrm{a}$ & $\mathrm{n} / \mathrm{a}$ \\
\hline Afghanistan & 2 & 2 & 100 & 3 & 2 & 2 & 100 & 3 \\
\hline Pakistan & 2 & 2 & 100 & 3 & 2 & 2 & 100 & 3 \\
\hline Australia & 2 & 2 & 100 & 12 & 1 & 1 & 100 & 10 \\
\hline Bangladesh & 2 & 2 & 100 & 16 & 0 & $\mathrm{n} / \mathrm{a}$ & $\mathrm{n} / \mathrm{a}$ & $\mathrm{n} / \mathrm{a}$ \\
\hline Solomon Islands & 2 & 1 & 50 & 15 & 1 & 0 & 0 & 0 \\
\hline Cambodia & 1 & 1 & 100 & 15 & 0 & $\mathrm{n} / \mathrm{a}$ & $\mathrm{n} / \mathrm{a}$ & $\mathrm{n} / \mathrm{a}$ \\
\hline Sri Lanka & 1 & 1 & 100 & 14 & 0 & $\mathrm{n} / \mathrm{a}$ & $\mathrm{n} / \mathrm{a}$ & $\mathrm{n} / \mathrm{a}$ \\
\hline
\end{tabular}

(Table 3, column 4), with the exceptions of China (72 \%), Papua New Guinea (64\%), Indonesia (55\%) and the Solomon Islands (50\%). For most countries the average number of $\mathrm{BGCl}$ records for 'all taxa' (calculated across only those taxa 'in cultivation') is 10 or more, showing that taxa 'in cultivation' are relatively secure. In contrast, Indonesia, the Philippines, Afghanistan and Pakistan have $\mathrm{BGCl}$ averages of three or fewer, indicating that those taxa are not secure 'in cultivation'.

For Red List taxa, 10 countries and 'Europe' have $100 \%$ of taxa 'in cultivation' (Table 3, column 8) although this is only 15 taxa in total, while a further seven countries have $75 \%$ or more of Red List taxa 'in cultivation'. In theory, Target 8 is met for these 17 countries and 'Europe' although some origins have low average numbers of BGCl records per taxon 'in cultivation', e.g., $79 \%$ of Red List taxa that originate in the Philippines are 'in cultivation', although the average of two BGCl records per taxon indicates limited holdings. The six remaining countries, which have less than $75 \%$ of Red List taxa 'in cultivation', are Japan (62 \%, average nine $\mathrm{BGCl}$ records per taxon 'in cultivation'), China (60\%, average nine records), Malaysia (59\%, average two records), Papua New Guinea (29\%, average three records), Indonesia (28\%, average two records) and Solomon Islands (0\%, average zero records).

\section{Ranking for 'not in cultivation' factors and 'not in cultivation' score}

The rankings for the 'not in cultivation' factors (Appendix 2) demonstrate that China ranks highest for number of Red List taxa 'not in cultivation' (178 taxa), followed by Indonesia (80 taxa) and Papua New Guinea (24). The same three countries, in the same order, also top the ranking for numbers of endemic Red List taxa 'not in cultivation' (168, 75 and 21 taxa, respectively). The ranking for percentage of Red List taxa 'not in cultivation' is led by Solomon Islands ( $100 \%$ of one taxon), followed by Indonesia (72 \%) and Papua New Guinea (71 \%). Bhutan ranks highest for percentage of endemic Red List taxa 'not in cultivation' (100\% of one taxon) followed by Indonesia (75\%) and Papua New Guinea (72\%).

China, Indonesia and Papua New Guinea lead the ranking for numbers of Data Deficient taxa 'not in cultivation' $(126,60$ and 19 taxa, respectively), and also the ranking for numbers of endemic Data Deficient taxa 'not in cultivation' (122, 57, 17, respectively). The Solomon Islands (100\% of one taxon) leads the ranking for percentage of Data Deficient taxa 'not in cultivation', followed by Indonesia (86 \%), Papua New Guinea (70 \%) and China (69 \%). For endemic Data Deficient taxa, Bhutan has the highest rank (100\% of one taxon) followed by Indonesia (89 \%) and China and Papua New Guinea (74 \% each). 
Table 4 Countries of origin of Rhododendron (Gibbs et al. 2011, Argent 2015) ranked by number of taxa: showing number of taxa, number of endemic taxa, number of Red List taxa, number of endemic Red List taxa for each origin, with the percentage of each category for which there is wild source (WS) material 'in cultivation'. Total number of taxa is 1215 ; however, column two will not sum to 1215 as many taxa have more than one origin. Total number of Red List taxa is 715 ; however, column six will not sum to 715 as many taxa have more than one origin.

\begin{tabular}{|c|c|c|c|c|c|c|c|c|}
\hline Country of origin & $\begin{array}{l}\text { No. of } \\
\text { taxa }\end{array}$ & $\begin{array}{l}\text { Percentage of } \\
\text { taxa for which } \\
\text { there } \\
\text { is WS material } \\
\text { 'in cultivation' }\end{array}$ & $\begin{array}{l}\text { No. of } \\
\text { taxa that } \\
\text { are endemic }\end{array}$ & $\begin{array}{l}\text { Percentage of } \\
\text { endemic taxa } \\
\text { for which there } \\
\text { is WS material } \\
\text { 'in cultivation' }\end{array}$ & $\begin{array}{l}\text { No. of } \\
\text { Red List } \\
\text { taxa }\end{array}$ & $\begin{array}{l}\text { Percentage of } \\
\text { Red List taxa } \\
\text { for which there } \\
\text { is WS material } \\
\text { 'in cultivation' }\end{array}$ & $\begin{array}{l}\text { No. of } \\
\text { Red List } \\
\text { taxa that } \\
\text { are endemic }\end{array}$ & $\begin{array}{l}\text { Percentage of } \\
\text { endemic Red List } \\
\text { taxa for which there } \\
\text { is WS material } \\
\text { 'in cultivation' }\end{array}$ \\
\hline China & 649 & 45 & 442 & 36 & 447 & 32 & 354 & 27 \\
\hline Indonesia & 229 & 49 & 168 & 39 & 111 & 22 & 101 & 21 \\
\hline Myanmar & 137 & 61 & 9 & 44 & 62 & 50 & 5 & 80 \\
\hline India & 107 & 71 & 14 & 36 & 45 & 60 & 11 & 36 \\
\hline Papua New Guinea & 100 & 60 & 64 & 55 & 34 & 27 & 29 & 24 \\
\hline Malaysia & 83 & 74 & 53 & 74 & 32 & 53 & 25 & 56 \\
\hline Japan & 74 & 42 & 58 & 33 & 26 & 19 & 25 & 20 \\
\hline Bhutan & 57 & 81 & 4 & 25 & 13 & 69 & 1 & 0 \\
\hline Vietnam & 40 & 58 & 10 & 30 & 22 & 55 & 7 & 29 \\
\hline Nepal & 34 & 88 & 3 & 67 & 4 & 100 & 2 & 100 \\
\hline Philippines & 33 & 79 & 30 & 77 & 19 & 74 & 19 & 74 \\
\hline USA & 29 & 38 & 21 & 38 & 8 & 0 & 7 & 0 \\
\hline Taiwan & 23 & 65 & 16 & 69 & 11 & 46 & 10 & 50 \\
\hline Russia: east & 15 & 47 & 3 & 0 & 1 & 100 & 1 & 100 \\
\hline Brunei & 12 & 83 & 0 & $\mathrm{n} / \mathrm{a}$ & 1 & 100 & 0 & $\mathrm{n} / \mathrm{a}$ \\
\hline Thailand & 12 & 83 & 1 & 100 & 1 & 100 & 1 & 100 \\
\hline Korea Sth & 11 & 91 & 2 & 50 & 0 & $\mathrm{n} / \mathrm{a}$ & $\mathrm{n} / \mathrm{a}$ & $\mathrm{n} / \mathrm{a}$ \\
\hline ‘Europe' aggregate & 10 & 90 & 8 & 88 & 2 & 100 & 2 & 100 \\
\hline Canada & 9 & 78 & 0 & $\mathrm{n} / \mathrm{a}$ & 1 & 0 & 0 & $\mathrm{n} / \mathrm{a}$ \\
\hline Korea Nth & 9 & 78 & 0 & $\mathrm{n} / \mathrm{a}$ & 0 & $\mathrm{n} / \mathrm{a}$ & $\mathrm{n} / \mathrm{a}$ & $\mathrm{n} / \mathrm{a}$ \\
\hline Laos & 7 & 100 & 0 & $\mathrm{n} / \mathrm{a}$ & 1 & 0 & 0 & $\mathrm{n} / \mathrm{a}$ \\
\hline Hong Kong & 6 & 67 & 0 & $\mathrm{n} / \mathrm{a}$ & 1 & 100 & 0 & $\mathrm{n} / \mathrm{a}$ \\
\hline Mongolia & 5 & 80 & 0 & $\mathrm{n} / \mathrm{a}$ & 0 & $\mathrm{n} / \mathrm{a}$ & $\mathrm{n} / \mathrm{a}$ & $\mathrm{n} / \mathrm{a}$ \\
\hline Greenland & 3 & 100 & 0 & $\mathrm{n} / \mathrm{a}$ & 0 & $\mathrm{n} / \mathrm{a}$ & $\mathrm{n} / \mathrm{a}$ & $\mathrm{n} / \mathrm{a}$ \\
\hline Solomon Islands & 2 & 50 & 0 & $\mathrm{n} / \mathrm{a}$ & 1 & 0 & 0 & $\mathrm{n} / \mathrm{a}$ \\
\hline Bangladesh & 2 & 100 & 0 & $\mathrm{n} / \mathrm{a}$ & 0 & $\mathrm{n} / \mathrm{a}$ & $\mathrm{n} / \mathrm{a}$ & $\mathrm{n} / \mathrm{a}$ \\
\hline Australia & 2 & 100 & 2 & 100 & 1 & 100 & 1 & 100 \\
\hline Afghanistan & 2 & 100 & 2 & 100 & 2 & 100 & 0 & $\mathrm{n} / \mathrm{a}$ \\
\hline Pakistan & 2 & 100 & 2 & 100 & 2 & 100 & 0 & $\mathrm{n} / \mathrm{a}$ \\
\hline Sri Lanka & 1 & 100 & 1 & 100 & 0 & $\mathrm{n} / \mathrm{a}$ & $\mathrm{n} / \mathrm{a}$ & $\mathrm{n} / \mathrm{a}$ \\
\hline Cambodia & 1 & 100 & 0 & $\mathrm{n} / \mathrm{a}$ & 0 & $\mathrm{n} / \mathrm{a}$ & $\mathrm{n} / \mathrm{a}$ & $\mathrm{n} / \mathrm{a}$ \\
\hline
\end{tabular}

The ninth factor in the 'not in cultivation' ranking, the average number of records per Red List taxon on the BGCl database (calculated across all taxa in the category, not just those in cultivation'), is led by Indonesia and Solomon Islands with a rounded average of zero. Of the nine countries for which the $\mathrm{BGCl}$ average is fewer than three records per taxon, four countries (Afghanistan, Pakistan, Solomon Islands and Canada) have relatively few taxa. Brunei has 12 taxa; however, they are all shared with Malaysia, while Indonesia, Malaysia, Papua New Guinea and the Philippines have greater numbers of taxa and thus represent the greater risk.

When the 'not in cultivation' score is calculated (Fig. 2), Red List taxa from Indonesia (127 points), China (119 points) and Papua New Guinea (118 points) have the weakest representation 'in cultivation'. Some countries, such as Myanmar and India, which have relatively high numbers of Red List taxa, are placed in the middle of this ranking because they have greater numbers of their taxa 'in cultivation' and fewer Data Deficient taxa. Countries at the lower end of this ranking (e.g., Laos, Brunei) tend to be those with lower numbers of Red List taxa, with few or none Data Deficient, and with most taxa 'in cultivation'.

\section{Total Score}

When countries of origin are ranked for Total Score (the sum of Red List score and 'not in cultivation' score) Indonesia has the highest score (281 points of a maximum of 303 ) and would therefore be considered the highest priority for ex situ conservation (Fig. 3). In second place is China (276 points), followed by Papua New Guinea (258 points).

\section{Wild-source material}

Percentage of wild-source (WS) material 'in cultivation' for each origin varies from $0 \%$ to $100 \%$ (Table 4), with endemic taxa tending to have more limited representation. (Overall, $69 \%$ of 'all taxa' and $59 \%$ of Red List taxa had wild-source material present 'in cultivation' (MacKay et al. 2017)). Origins with the lowest percentages of wild-source material for Red List taxa 'in cultivation' are the one-taxon countries (Solomon Islands, Laos and Canada) and the eight taxa from the USA. Low percentages of wild-source material are also seen for Japan (19\% of Red List taxa 'in cultivation'), Indonesia (22\%), Papua New Guinea (27\%), China (32\%) and Taiwan (46\%). For all other countries, $50 \%$ or more of Red List taxa 'in cultivation' have wild-source material present. For endemic Red List taxa, the pattern is similar with the poorest representation being Bhutan and the USA (0\%), Japan (20\%), Indonesia (21\%), Papua New Guinea (24\%), China (27\%), Vietnam (29\%) and India (36\%). The remaining origins have $50 \%$ or more of endemic Red List taxa represented 'in cultivation' by wild-source material. 


\section{Additional taxa}

While undertaking the present analysis we recorded 153 valid taxa that are yet to be Red List evaluated. Although Argent (2015) completed the evaluations for all but four vireya taxa, another 149 taxa from the remaining subgenera were not evaluated by Gibbs et al. (2011). When organised by country of origin (Appendix 3) 96 of the additional taxa originate in China (84 endemic), with 20 from Japan (19 endemic), 11 from Myanmar (2 endemic) and less than nine from each of the other countries. The majority of the additional taxa (100 of 153) are 'in cultivation' (data not shown).

\section{DISCUSSION}

\section{Conservation issues and priorities}

In the present study we have analysed the occurrence of the 715 Red List taxa of Rhododendron by country of origin, and determined the incidence of taxa 'in cultivation' and 'not in cultivation' by country of origin, to identify Indonesia and China as priority countries of origin for conservation of this genus. Our results have also highlighted a number of conservation issues. Data on origin and endemism identify countries with shared taxa, where cooperation among countries would be desirable in a conservation programme, e.g., Nepal, India, Bhutan and China. Conversely, countries with high degrees of endemism are also identified, e.g., the Philippines and Japan where only limited inter-country communities of interest will be possible, highlighting the desirability of a strong within-country collections network and effective relationships with international collections.

Data on Red List taxa demonstrate that for some countries endemic taxa are more threatened than non-endemic taxa, while for other countries the reverse is true. Comparison among endemic taxa demonstrates that there is greater conservation urgency for some countries (e.g., endemic taxa from India and Vietnam) than for others (e.g., Myanmar and Bhutan). Prioritising endemic taxa is a recognised conservation approach (Powledge 2011, Cavendar et al. 2015), with our analysis showing that a finer resolution is possible and comparisons can also be made among countries.

The Red List analysis (Fig. 1) identifies China, Indonesia and Papua New Guinea as highest priority countries and, as this ranking excludes any cultivation factors, the priority applies to both in situ and ex situ conservation. While China and Indonesia might be expected to take priority for conservation simply due to high numbers of taxa, they also rank highly because of the extent of endemism and Data Deficiency. The same two factors result in Papua New Guinea and the Philippines ranking above other origins (Myanmar, India) which have more Red List taxa in the first instance.

The Total Score analysis (Fig. 3), where cultivation factors are taken into account, prioritises Indonesia, China and Papua New Guinea for ex situ conservation. While China has a higher Red List score than Indonesia, and China would take priority for in situ conservation, taxa from Indonesia have poorer representation 'in cultivation' and so take priority for ex situ conservation. (Notably, there is limited wild-source material 'in cultivation' for the countries prioritised by Total Score, exacerbating the ex situ conservation challenge for taxa from those countries.) In the middle of the ranking are countries which may have reasonable numbers of Red List taxa (e.g., Myanmar, Bhutan, Taiwan and the USA); however, factors such as good representation 'in cultivation' or lesser degrees of Data Deficiency place them in the middle of the ranking. At the lower end of the ranking are several countries which tend to have smaller numbers of taxa, fewer endemic taxa, most taxa 'in cultivation' and relatively few Data Deficient taxa.

Data Deficiency is a recurring issue in our analysis. Sixty three percent of Red List Indonesian taxa are rated Data Deficient and Rahman (2008) noted that Data Deficiency was most acute for non-Java species. Data Deficiency is a lesser problem for taxa of Chinese origin, where $41 \%$ of Red List taxa were rated Data Deficient (although the number of taxa is more than twice that of Indonesia), indicating better knowledge of taxa from China and the capacity to assign a Red List category rather than being obliged to use the Data Deficient category. For several countries $100 \%$ of their Data Deficient taxa are endemic, although urgency for conservation action would be greatest for Japan and the Philippines, as they have the greatest numbers of taxa.

The Data Deficient taxa comprise three broad groups. The first group is about 112 taxa for which Gibbs et al. (2011), despite using some 60 references, were unable to provide any information. The second group is about 90 taxa listed by Gibbs et al. (2011) which are only known from the type specimen or a very limited number of specimens. Often this is a reflection of limited field studies; however, the issue can also be taxonomic. In the third group are about 100 taxa for which Gibbs et al. (2011) indicate that taxonomic status is uncertain or subject to debate; issues include taxa that are poorly described, suspected to be hybrids, or where there is debate about their synonymy with another taxon. These issues often coincide, e.g., few specimens were collected because the taxon is a hybrid. Such difficulties are part of the 'big genus' characteristics of Rhododendron (Frodin 2004) and highlight the importance of using DNA-based methods to enhance our scientific consideration of conservation priorities.

Biogeography is another issue raised by the present results. Although one of the five top-ranked countries for Total Score is located on mainland Asia (China), the next four are located on nearby island groups in Japan and Southeast Asia (Indonesia, Malaysia, the Philippines and Papua New Guinea). In our study on subg. Vireya (MacKay et al. 2016), we determined that taxa from New Guinea (Indonesia and Papua New Guinea) should be prioritised over taxa from Borneo (Malaysia, Indonesia and Brunei); however, we did not consider the islands that comprise Japan or separate out the many small islands that surround the larger islands of Southeast Asia. The extent to which any individual island is important for Rhododendron conservation is yet to be determined and should be the subject of further research.

Finally, our analysis shows that 17 countries and 'Europe' have met the $75 \%$ of Red List taxa 'in cultivation' required for Target 8 , although low average numbers of BGCI records per taxon is a key issue (suggesting that genetic representation is likely to be poor). (Unfortunately this problem is common in plant conservation in general, e.g., $33 \%$ and about $50 \%$ of threatened species were present in only one collection (Pritchard et al. 2011, Cires et al. 2013, Hird \& Kramer 2013).) Conversely, six countries do not meet the $75 \%$ requirement for Target 8 . Three (China, Japan, Malaysia) are reasonably well placed to achieve $75 \%$ in the near future; however, there is clearly some work to do with respect to Papua New Guinea, Indonesia and the Solomon Islands in terms of both the range of taxa 'in cultivation' and the numbers of each taxon held.

\section{Assumptions and limitations}

The key assumption of the present analysis is that the primary drivers for ex situ conservation are threat, endemism, Data Deficiency, and presence 'in cultivation' (Newton \& Oldfield 2008, Powledge 2011, Sharrock et al. 2014, Cavendar et al. 2015).

The first limitation of the present study relates to the two key data elements of a geographic analysis for ex situ conservation, 
an accurate Red List and accurate origin data, and these elements have some unavoidable limitations. Acquiring and organising up-to-date data for a Red List assessment can be difficult in general (Newton \& Oldfield 2008, Oldfield 2010, Blackmore et al. 2011, Cires et al. 2013) and this weakness has been noted in particular for Rhododendron (Ma et al. 2014, Rahman \& Rozak 2016). Gibbs et al. (2011) recognised this issue and noted the need for additional field research that may modify an assessment. For example, Ma et al. (2014) increased the threat level of two Rhododendron species while Rahman \& Rozak (2016) reduced the threat level of two other species on the basis of additional field research. Despite the possible weaknesses in the Rhododendron Red List, conservation planning 'has to start somewhere' and the current Red List is a significant resource that can be used to inform future research. In due course, as new Red List assessments are made or previous assessments updated, these can be used in future conservation planning. The second key element of a geographic analysis is origin data. This is primarily obtained from type specimens, herbarium records and field studies; however, these are not always comprehensive and distribution can sometimes be defined by collecting patterns rather than actual physical distribution. Again, field work can revise distribution data (e.g., Huong \& Hiep 2012, Ma et al. 2013, Yang et al. 2015); however, in large genera like Rhododendron the sheer size of the task is challenging and there can be difficulties obtaining expertise, resources and access for field studies (Gibbs et al. 2011).

The next limitation relates to the 'cultivation' analyses. Because of its size and scope, the BGCl database was used as a primary source of data on taxa in cultivation; subsequently data on collections at the Royal Botanic Gardens Kew and Edinburgh, and in New Zealand, were added as prior studies had shown these to contain significant collections (BGCl 2012, MacKay \& Gardiner 2017, MacKay et al. 2017). However, while the aforementioned sources are extensive, and some are readily accessible through online databases, there are other notable Rhododendron collections world-wide. Large private collections in the United Kingdom, the USA and Europe were not included in the BGCl (2012) study, and there were only 11 gardens from China and Asia among the sites considered. Additional research should be undertaken to expand the range of collections used to describe the 'in cultivation' and 'not in cultivation' aspects of any future analyses. Expansion of the range of collections should also overcome the apparent lack of wild-source material for North American taxa. As our main comparison was with British collections, which may not prioritise North American taxa, and the $\mathrm{BGCl}$ records do not indicate wild-source, it is likely that the absence of wild-source North American material is an anomaly that will disappear when a wider range of collections is examined.

The final limitation relates to the additional taxa that have not yet been Red List assessed. As only three of the 153 additional taxa come from Indonesia (first ranked in Total Score) and 96 come from China (second ranked for Total Score) with 84 endemic, a re-run of our analysis (when Red List assessments for the additional taxa become available) may create a different ranking. Similarly, the rankings may be changed by newly discovered species such as $R$. stanleyi S. James \& Argent (James \& Argent 2017 ) and yet to be described species, as indicated by more than 40 wild-collected aff. taxa listed at Edinburgh (Catalogue of the Living collections: http://elmer.rbge.org.uk/bgbase/livcol/ bgbaselivcol.php; acc. 11 Jan. 2017).

\section{Conservation action}

Our analysis shows that Rhododendron taxa from Indonesia and China should take priority for ex situ conservation. This should take place within an overall conservation framework (ex situ and in situ) and in relation to 'all flora' for each country of origin. Indonesia held $21.5 \%$ of Red List flora in general in botanic gardens in 2010 (Purnomo et al. 2010), with the present results showing Red List Rhododendron of Indonesian origin are similarly placed with $28 \%$ 'in cultivation' (but not necessarily in Indonesia). Rhododendron taxa of Chinese origin (69\% of taxa Red Listed) are in a worse position than Chinese flora in general, where $20 \%$ are considered at risk (Huang et al. 2015). Although China has in place a conservation strategy and a range of in situ and ex situ initiatives (Lopez-Pujol et al. 2011, Ma et al. 2013, Huang et al. 2015, 2016), more ex situ collections are needed in general (Raven 2011, Huang 2015), and more field work and ex situ collections are needed for Rhododendron in particular (Ma et al. 2013, 2014). Other countries also have in situ conservation initiatives for 'all flora', e.g., Nepal (IUCN Nepal 2010) and India (Rana \& Samant 2010), with initiatives that are particular to Rhododendron (e.g., Singh et al. 2009). Individual countries will wish to determine their own conservation priorities and the place of Rhododendron among those priorities is as yet unknown for many countries. For Rhododendron specifically, we have already proposed actions and priorities for ex situ conservation in relation to subgenera (MacKay \& Gardiner 2017) and subg. Vireya (MacKay et al. 2016), and many of the underlying principles will also apply to the geographic analysis performed in the present study. The following actions for ex situ conservation are recommended:

- Individual nations that are a country of origin for Rhododendron should, where possible and where resources allow:

- Primarily focus their ex situ collections on their own endemic taxa, particularly Red List endemic taxa;

- Form 'communities of interest' with associated countries in relation to non-endemic taxa. While individual countries may wish to perform their own field studies, research into habitat, taxonomy, and genetic diversity (including DNA-based studies) may be useful areas of cooperation;

- Develop a programme of field work to:

i. acquire more accessions of taxa that have limited representation in cultivation;

ii. investigate relevant aspects of any taxon, with first priority assigned to Critically Endangered taxa;

iii. investigate Data Deficient taxa, to clarify their conservation status; and

iv. investigate taxa that are taxonomically uncertain; and

- Develop additional ex situ collections, preferably in a botanic garden that is compatible with the habitat of origin or within a similar climate zone (while also selecting locations that minimise the risk of hybridisation between plants held in the collection and those in nearby native vegetation).

- Nations that are not a country of origin for Rhododendron should, where possible and where resources allow:

- Focus existing ex situ collections on endemic taxa and the most endangered taxa from priority origins, prioritising those origins where there are currently few ex situ collections;

- Propagate and disperse existing accessions of priority taxa, to other ex situ collections to guard against loss, particularly taxa of wild-source origin;

- Consider becoming the designated primary or duplicate collection for selected groups of taxa, depending on climate zone, resources, and the characteristics of the existing collection; and

- Contribute expertise and participate in activities to acquire new ex situ material and develop new collections. 
The actions above should be supported by a programme of international coordination and cooperation for matters such as:

i. determining where primary and duplicate collections would be located;

ii. exchanging wild-source material among collections (where possible);

iii. expanding the ex situ analysis to gain a broader coverage of taxa 'in cultivation'; and

iv. investigate the diversity of existing collections and develop protocols to manage diversity and avoid genetic drift.

We have already proposed some elements of an international programme of cooperation (MacKay et al. 2016, MacKay \& Gardiner 2017), and the results of the present analysis will enable priority countries of origin to be added to the international framework.

\section{CONCLUSION}

The present study has shown that taxa that originate in Indonesia, China and Papua New Guinea are the highest priority for ex situ conservation of Rhododendron, while the priority for in situ conservation should be taxa from China, Indonesia and Papua New Guinea. Target 8 has been achieved for 17 countries and 'Europe' in terms of the percentage of taxa 'in cultivation' although holdings of some taxa are limited and genetic diversity is likely to be low. Compared to plants in general, Rhododendron has a high percentage of taxa 'in cultivation' and conservationists will be relatively well placed to undertake further developments in ex situ conservation. While individual countries will wish to determine their own conservation priorities, and endemic taxa are clearly important, there are also communities of interest where taxa are shared among countries and where joint conservation initiatives may be possible. Given the size of the genus Rhododendron, its taxonomic complexity, and the range of habitats and climate zones it occupies, development of an international and coordinated ex situ strategy is a subject for on-going research.

Acknowledgements This study has sourced data from several online databases and we appreciate the opportunity to access those databases. We also thank New Zealand collection holders who contributed data to this study, and are grateful to the New Zealand Rhododendron Association, Pukeiti Rhododendron Trust, Taranaki Regional Council, The New Zealand Institute for Plant \& Food Research Limited, and Massey University for supporting this research.

\section{REFERENCES}

Argent G. 2015. Rhododendron of the subgenus Vireya. 2nd edition. Royal Botanic Garden, Edinburgh, in association with The Royal Horticultural Society, UK.

Baird R, Wood-Jones A, Varco J, et al. 2014. Rhododendron decline in the Great Smokey Mountains and surrounding areas: intensive study of biotic and abiotic parameters associated with the decline. Southeastern Naturalist 13, 1: 1-25.

Bharali S, Paul A, Khan ML. 2014. Population status of Rhododendron mechukae - A newly recorded endemic species from Eastern Himalaya. Journal of Biodiversity Management and Forestry 2, 3: 5-10. doi: http:// dx.doi.org/10.4172/2327-4417.1000113.

Bharali S, Paul A, Khan ML, et al. 2011. Species diversity and community structure of a temperate mixed Rhododendron forest along an altitudinal gradient in west Siang district of Arunachal Pradesh, India. Nature and Science 9, 12: 125-140.

BGCl. 2012. Global survey of ex situ Rhododendron collections. Botanic Gardens Conservation International, (Richmond) UK. https://www.bgci.org/ plant-conservation/rhododendron_survey/. Last accessed 11 Aug. 2017.

Blackmore S, Gibby M, Rae D. 2011. Strengthening the scientific contribution of botanic gardens to the second phase of the Global Strategy for Plant Conservation. Botanical Journal of the Linnaean Society 166: 267-281.
Castaneda-Alvarez NP, De Hann S, Juarez H, et al. 2015. Ex situ conservation priorities for the wild relatives of potato (Solanum L., section Petota). PLoS ONE 10, 4: e0122599. doi: http://dx.doi.org/10.1371/journal.pone.0122599.

Cavendar N, Westwood M, Bectholdt C, et al. 2015. Strengthening the conservation value of ex situ tree collections. Oryx July 2015: 1-9.

Chamberlain D, Hyam R, Argent G, et al. 1996. The genus Rhododendron: its classification and synonymy. Royal Botanic Garden Edinburgh, Edinburgh, UK.

Cires E, De Smet Y, Cuesta C, et al. 2013. Gap analysis to support ex situ conservation of genetic diversity in Magnolia, a flagship group. Biodiversity Conservation 22: 567-590.

Cox PA, Cox KNE. 1997. The encyclopedia of Rhododendron species. Glendoick Publishing, Glencarse, Scotland.

Cox PA, Hutchison P. 2008. Seeds of adventure. In search of plants. Garden Art Press, Suffolk, UK.

Ennos RA, French GC, Hollingsworth PM. 2005. Conserving taxonomic complexity. Trends in Ecology and Evolution 20, 4: 164-168.

Ennos RA, Whitlock R, Fay MF, et al. 2012. Process-based species action plans: an approach to conserve contemporary evolutionary processes that sustain diversity in taxonomically diverse groups. Botanical Journal of the Linnaean Society 168: 194-203.

Ensslin A, Tschope O, Burkhart M, et al. 2015. Fitness decline and adaptation to novel environments in ex situ plant collections: current knowledge and future perspectives. Biological Conservation 192: 394-401.

Fang MY, Fang RC, He MY, et al. 2005. Rhododendron. Flora of China 14: 260-455.

Farnsworth EJ, Klionsky S, Brumback WE, et al. 2006. A set of simple decision matrices for prioritising collection of rare plant species for ex situ conservation. Biological Conservation 128: 1-12.

Frodin DG. 2004. History and concepts of big plant genera. Taxon 53, 3: 753-776.

Gibbs D, Chamberlain D, Argent G. 2011. The red list of Rhododendrons. Botanic Gardens Conservation International, Richmond, UK. https://www. bgci.org/plant-conservation/rhododendron_red_list/. Last accessed 11 Aug. 2017.

Goodall-Copestake WP, Hollingsworth ML, Hollingsworth PM, et al. 2005. Molecular markers and ex situ conservation of the European elms (UImus ssp.). Biological Conservation 122: 537-546.

Heywood V[H]. 2009. Botanic gardens and genetic conservation. Sibbaldia: The Journal of Botanic Garden Horticulture 7: 5-18.

Heywood VH. 2015. In situ conservation of plant species - an unattainable goal? Israel Journal of Plant Sciences 2015: 1-21. doi: http://dx.doi.org/1 0.1080/07929978.2015.1035605.

Hird A. 2012. A quarter-century perspective on the center for plant conservation collections at the Arnold arboretum. Arnoldia 70, 1: 11-25.

Hird A, Kramer A. 2013. Achieving Target 8 of the Global Strategy for Plant Conservation: lessons learned from the North American collections assessment. Annals of the Missouri Botanical Garden 99, 2: 161-166.

Huang HW. 2015. Prospects for the future. In: Hong D, Blackmore S, A companion to the Flora of China. 2nd ed: 446-452. Cambridge University Press, UK.

Huang HW, Wyse-Jackson PS, Chen LZ. 2015. Conservation strategies. In: Hong D, Blackmore S, A companion to the Flora of China. 2nd ed: 418-445. Cambridge University Press, UK.

Huang J, Huang J, Lu X, et al. 2016. Diversity distribution patterns of endemic seed plant species and their implications for conservation planning. Scientific Reports 6:33913. doi: http://doi.org/10.1038/srep33913.

Huong NTT, Hiep NT. 2012. New record of two species of Rhododendron L. (Ericaceae) for the flora of Vietnam. Tap Chin Sinh Hoc 34, 4: 446-451. http://vjs.ac.vn/index.php/vjbio/article/view/2680. Last accessed $10 \mathrm{Apr}$. 2017.

IUCN Nepal. 2010. Tinjure-Milke-Jaljale Rhododendron conservation area: a strategy for sustainable development. IUCN Nepal Country Office, Kathmandu. http://cmsdata.iucn.org/downloads/tinjure_milke_jaljale_rhododendron_conservation_area__a_strategy_for_sustainable_dev.pdf. Last accessed 13 Feb. 2017.

Jalli R, Aravind J, Pandey A. 2015. Conservation and management of endemic and threatened plant species in India: an overview. Plant Biology and Biotechnology Springer, India 2015: 461-486. doi: https://doi. org/10.1007/978-81-322-2283-5_24.

James SA, Argent G. 2017. Rhododendron stanleyi S.James \& Argent: a new Rhododendron species (Ericaceae) from Papua New Guinea. Edinburgh Journal of Botany. doi: https://doi.org/10.1017/S096042861700004X.

Kozlowski G, Gibbs D, Huan F, et al. 2012. Conservation of threatened relict trees through living ex situ collections: lessons from the global survey of the genus Zelkova (Ulmaceae). Biodiversity Conservation 21: 671-685. 
Krupnick GA, Kress WJ. 2003. Hotspots and ecoregions: a test of conservation priorities using taxonomic data. Biodiversity and Conservation 12: 2237-2253.

Leslie AC. 2004. The International Rhododendron register and checklist. 2nd ed. Royal Horticultural Society, UK.

Liu JQ, Ren MX, Susanna A, et al. 2015. Special issue on ecology evolution and conservation of plants in China: introduction and some considerations. Collectanea Botanica 34: e001. doi: http://dx.doi.org/10.3989/ collectbot.2015.v34.001.

Lopez-Pujol J, Wang HF, Zhang ZY. 2011. Conservation of Chinese plant diversity: an overview. Ch 9. In: Pavlinov E (ed), Research in biodiversity models and applications. InTech. doi: http://dx.doi.org/10.5772/24395. https://www.intechopen.com/books/research-in-biodiversity-models-andapplications/conservation-of-chinese-plant-diversity-an-overview. Last accessed 16 May 2017

Lopez-Pujol J, Zhang FM, Ge S. 2006. Plant biodiversity in China: richly varied, endangered, and in need of conservation. Biodiversity and Conservation 15: 3983-4026.

Lowe RAW. 1988. The National Council for the Conservation of Plants and Gardens (NCCPG). Royal New Zealand Institute of Horticulture Annual Journal 15: 29-31.

Ma Y, Chen G, Grumbine RE, et al. 2013. Conserving plant species with extremely small populations (PSESP) in China. Biodiversity Conservation 22: 803-809.

Ma Y, Nielsen J, Chamberlain DF, et al. 2014. The conservation of Rhododendrons is of greater urgency than has been previously acknowledged in China. Biodiversity Conservation 23: 3149-3154.

MacKay M[B], Gardiner SE. 2017. A model for determining ex situ conservation priorities in big genera is provided by analysis of the subgenera of Rhododendron (Ericaceae). Biodiversity and Conservation 26: 189-208. doi: https://doi.org/10.1007/s10531-016-1237-0.

MacKay M[B], Smith G, Gardiner SE. 2016. Analysis of geographic and taxonomic groups informs conservation of Rhododendron subgenus Vireya (Ericaceae). Blumea 61: 170-180. doi: https://doi.org/10.3767/ $000651916 \times 693275$.

MacKay MB, Smith GF, Gardiner SE. 2017. New Zealand collections of Rhododendron L. as a resource for ex situ conservation. New Zealand Journal of Botany. doi: http://dx.doi.org/10.1080/0028825X.2017.1291434.

Mao AA, Gogoi R. 2012. Rhododendrons of Manipur and Nagaland, India. NeBio 3, 1: 1-10.

Maren IE, Vetaas OR. 2007. Does regulated land use allow regeneration of keystone forest species in the Annapurna conservation area, central Himalaya? Mountain Research and Development 27, 4: 345-351.

Melick DR, Kinch JP, Govan H. 2012. How global biodiversity targets risk becoming counterproductive: the case of Papua New Guinea. Conservation and Society 10, 4: 344-353.

Newton A, Oldfield S. 2008. Red listing the world's tree species: a review of recent progress. Endangered Species Research 6: 137-147.

Oldfield S. 2010. Plant conservation: facing tough choices. BioScience 60, 10: $778-779$.

Paudel PK, Bhattarai BP, Kindlmann P. 2012. An overview of the biodiversity in Nepal. In: Kindlmann P (ed), Himalayan biodiversity in the changing world: 1-40. Springer, The Netherlands. doi: https://doi.org/10.1007/97894-007-1802-9_1.

Paul A, Khan ML, Arunachalam A, et al. 2005. Biodiversity and conservation of rhododendrons in Arunchal Pradesh in the Indo-Burma biodiversity hotspot. Current Science 89, 4: 623-634.

Popescu R, Kopp B. 2013. The genus Rhododendron: an ethnopharmacological and toxicological review. Journal of Ethnopharmacology 147: 43-62.

Powledge F. 2011. The evolving role of botanical gardens. BioScience 61, 10: $743-749$.
Pradhan BK, Dabal S, Nilson J, et al. 2015. A note on Rhododendron mekongense - a new species record from Sikkim Himalaya, India. Journa American Rhododendron Society (Spring 2015): 76-80.

Pritchard DJ, Fa JE, Oldfield S, et al. 2011. Bring the captive closer to the wild: redefining the role of ex situ conservation. Oryx 46, 1: 18-23.

Purnomo DW, Hendrian R, Witono JR, et al. 2010. Indonesia botanic gardens achievement on Target 8 of the Global Strategy for Plant Conservation (GSPC). Buletin Kebun Raya 13, 2: 40-50.

Rae D. 2011. Fit for purpose: the importance of quality standards in the cultivation and use of live plant collections for conservation. Biodiversity Conservation 20: 241-258.

Rahman W. 2008. Data deficiency, taxonomical status and natural hybrid in the conservation of Rhododendron ssp. in Indonesia. Buletin Kebun Raya Indonesia 11, 2: 5-14.

Rahman W. 2015. Criteria for determining the priority species of threatened Rhododendron ssp. for ex situ conservation in Indonesia. Buletin Kuba Raya 18, 1: 31-40.

Rahman W, Rozak AH. 2016. Population size of two endangered Vireya Rhododendron species and its surrounding vegetation on the top of $\mathrm{Mt}$. Rantemario, Sulawesi. Buletin Kebu Raya 19, 1: 57-66.

Rana MS, Samant SS. 2010. Threat categorisation and conservation prioritisation of floristic diversity in the Indian Himalayan region: a state of art approach form Manali Wildlife Sanctuary. Journal for Nature Conservation 18: 159-168.

Ranjitkar S, Sujakhu NM, Jati R, et al. 2014. Yield and household consumption of Rhododendron arboreum as a fuelwood species in eastern Nepal. Biomass and Bioenergy 61: 245-253.

Raven PH. 2011. Plant conservation in the future: new challenges, new opportunities. Plant Diversity and Resources 33, 1: 1-9.

Samain M, Vanderschaeve L, Chaerle P, et al. 2009. Is morphology telling the truth about the evolution of the species rich genus Peperomia (Piperaceae)? Plant Systematics and Evolution 278: 1-21.

Sharrock S. 2012. Global strategy for plant conservation. A guide to the GSPC. Botanic Gardens Conservation International, Richmond, UK.

Sharrock S, Oldfield S, Wilson O. 2014. Plant conservation report 2014: A review of progress in implementation of the Global Strategy for Plant Conservation 2011-2020. Technical Series No. 81. Secretariat of the Convention on Biological Diversity, Montréal, Canada and Botanic Gardens Conservation International, Richmond, UK.

Shi JP, Zhu H. 2009. Tree species composition and diversity of tropical mountain cloud forest in the Yunnan, southwest China. Ecological Restoration 24: 83-92.

Singh KK, Rai LK, Gurung B. 2009. Conservation of rhododendrons in Sikkim Himalaya: an overview. World Journal of Agricultural Sciences 5, 3: 284-296.

Van der Ent A, Repin R, Sugau J, et al. 2015. Plant diversity and ecology of ultramafic outcrops in Sabah (Malaysia). Australian Journal of Botany 63: 204-215.

Van Welzen PC, Slik JWF, Alahuhuta J. 2005. Plant distribution patterns and plate tectonics in Malesia. In: Friis I, Balslev H, Plant diversity and complexity patterns: local, regional and global dimensions: 199-217. Proceedings of an international symposium held at the Royal Danish Academy of Sciences and Letters, Copenhagen 25-28 May 2003.

Webb CO, Ree R. 2012. Historical biogeography inference in Malesia. In: Gower D (ed), Biotic evolution and environmental change in Southeast Asia: 191-215. Cambridge University Press, UK.

Yang JC, Kwon YH, Ji SJ, et al. 2015. A new record of Rhododendron keiskei Miq. var. hypoglaucum Suto \& Suzuki (Ericaceae) in Korea. Korean Journal of Plant Taxonomy 45, 3: 239-242. 


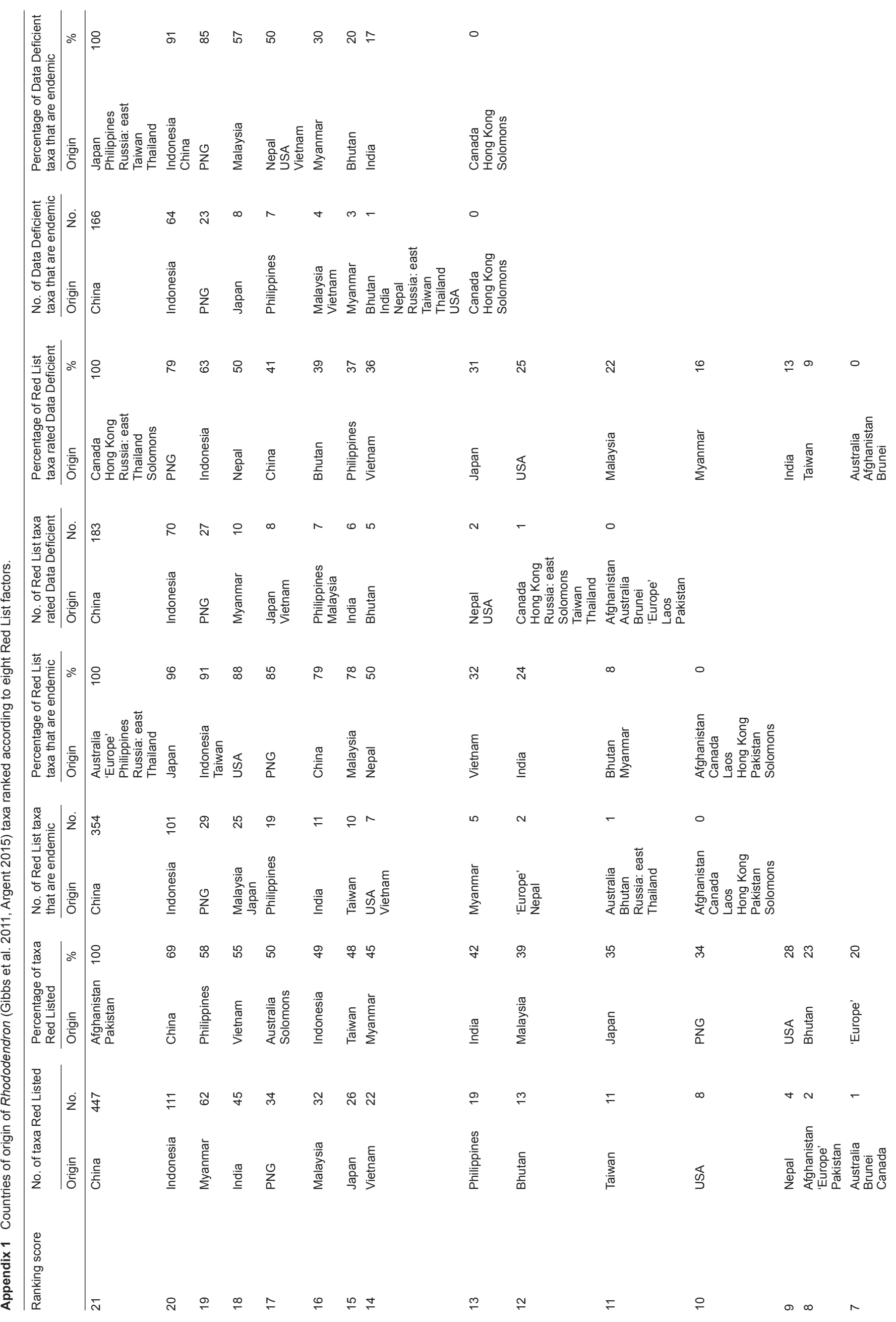




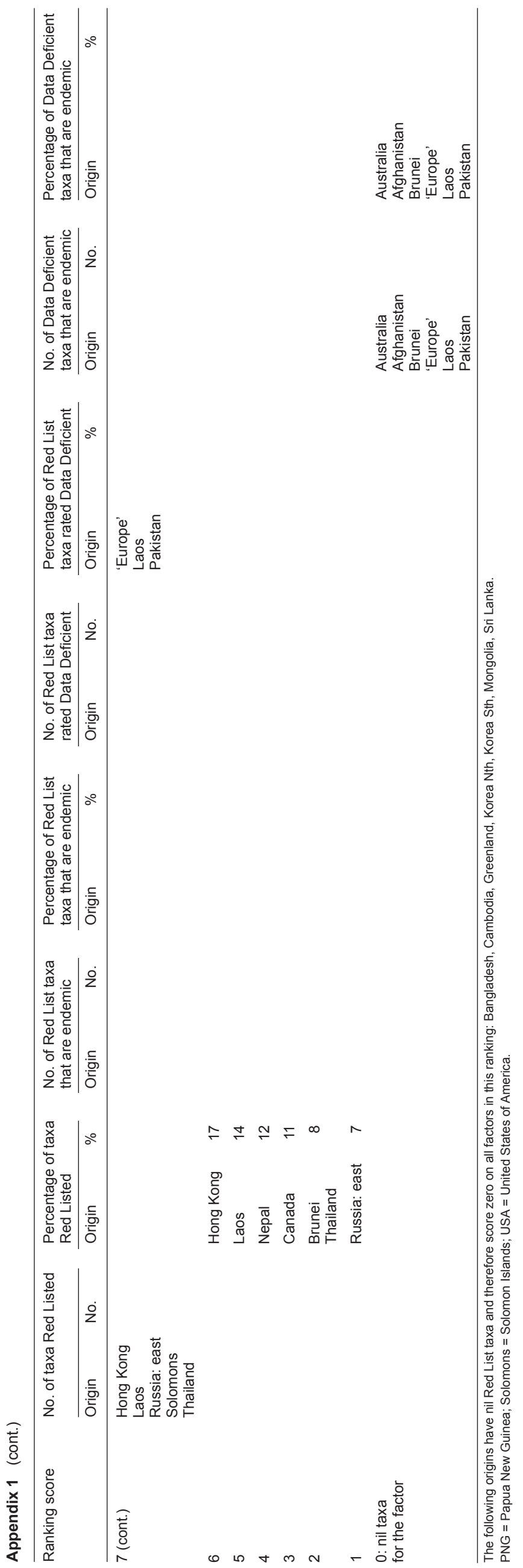




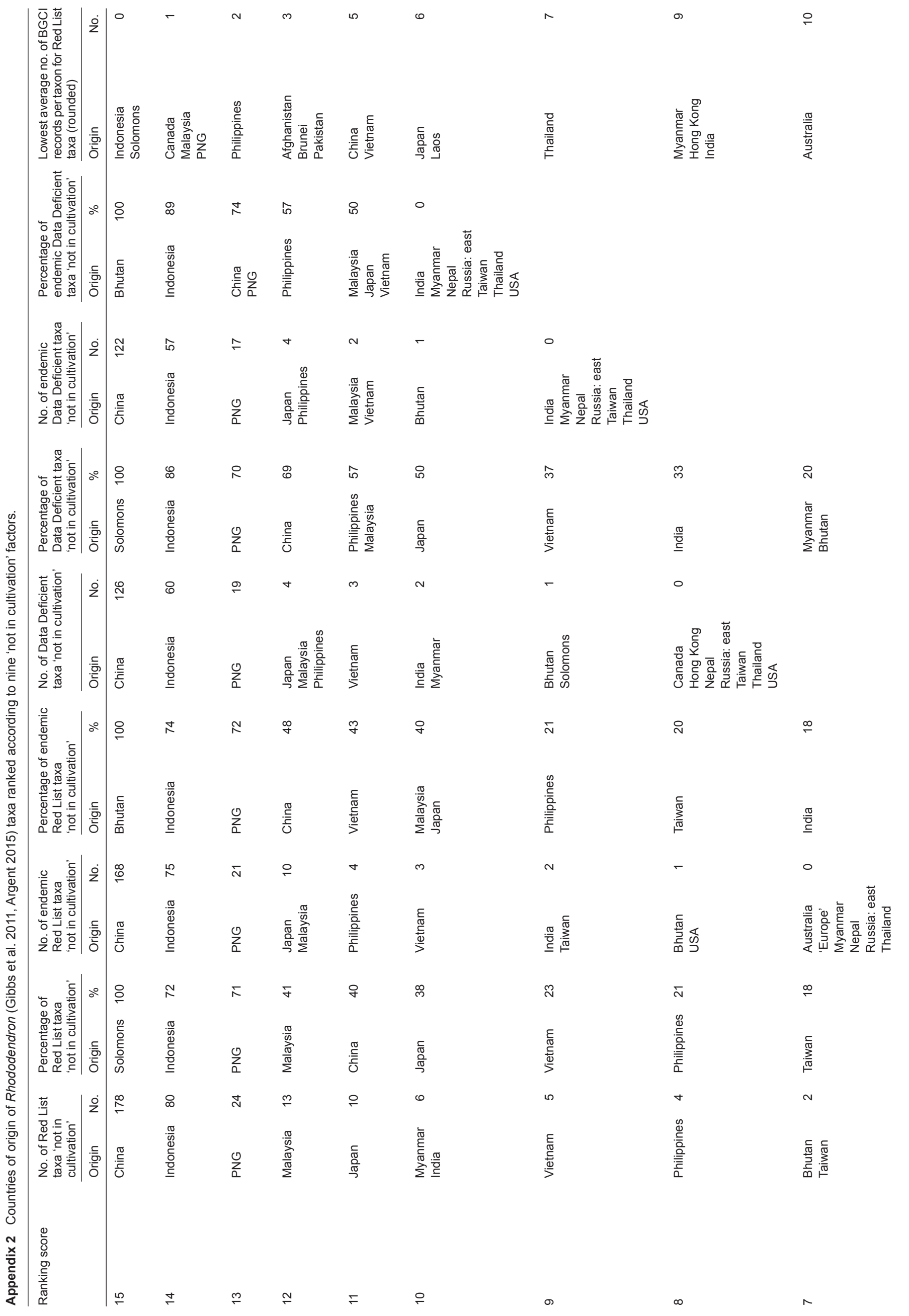




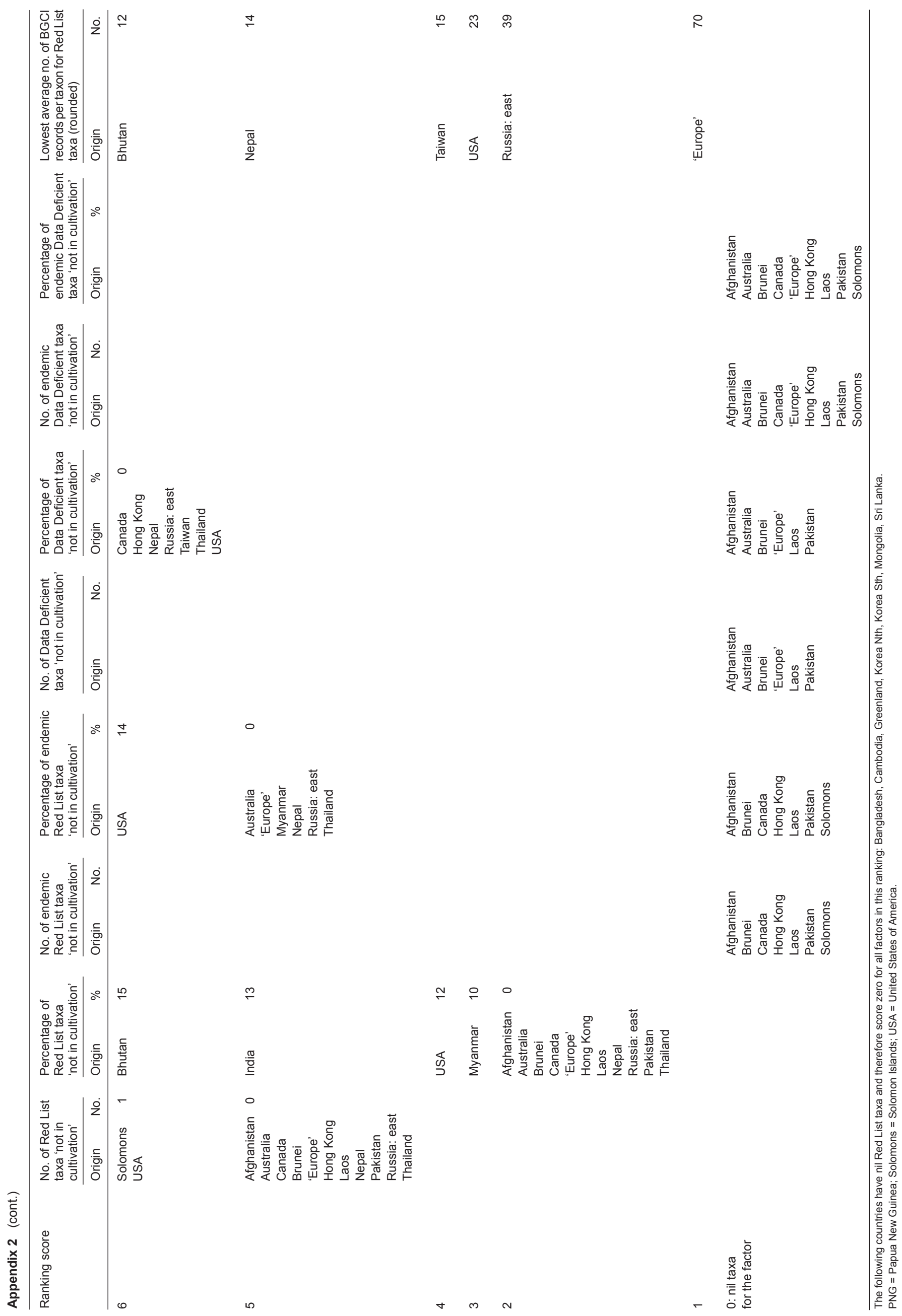


Appendix 3 Countries of origin of 153 Rhododendron taxa recorded in this research which are additional to those considered by Gibbs et al. (2011) and Argent (2015). The numbers of taxa in column two will not sum to 153 as some taxa have more than one origin.

\begin{tabular}{lcc}
\hline Country of origin & Total no. of taxa & No. of taxa that are endemic \\
\hline China & 96 & 84 \\
Japan & 20 & 19 \\
Myanmar & 11 & 2 \\
India & 9 & 4 \\
Vietnam & 6 & 2 \\
USA & 5 & 4 \\
Nepal & 5 & 3 \\
Bhutan & 5 & 3 \\
Taiwan & 4 & 4 \\
Malaysia & 3 & 3 \\
Indonesia & 3 & 0 \\
Russia: east & 3 & 1 \\
Korea Nth & 1 & 0 \\
Korea Sth & 1 & 0 \\
Papua New Guinea & 1 & 0 \\
'Europe' aggregate & 1 & 0 \\
\hline
\end{tabular}

